Sir G. G.: Trans. Cambridge Phil. Soc. (II), 9, 8. (1856) [also, Math. and Phys. Paper; 3,1 (1901)]. - [35] Kunnen, F., and Ph. Furtwänglar: Bestimmung der absoluten Größe der Schwerkraft zu Potsdam. Veröff. königl. Preuss. geod. Inst. 1906, 27. - [36] Helmert, F. R.: Beiträge zur Theorie des Reversionspendels. Veröff. Preuss. geod. Inst. 1898. - [37] Borda; I. C.: Base du Système Métrique Décimal. Vol. 3, Paris 1810. - [38] Dryden, H. L.: J. Research Nat. Bur. Standards 29, 303 (1942). - [39] GoILlet, A.: C. R. Acad. Sci.
(Paris) 165, 1050 (1917). - [40] GuILLET, A.: C. R. Acad. Sci. (Paris) 207, 614 (1938). - [41] Volet, C.: C. R. Acad. Sci. (Paris) 222, 373 (1946). - [42] Voler, C.: C. R. Acad. Sci. (Paris) 224, 1815 (1947). - [43] IvanoFr, A.: Bull. géod. 52, 481 (1936). - [44] Woon, R. W.: Astrophys. J. 29, 164 (1909).

\section{A. H. Cook}

Standards Division National Physical Laboratory Teddington, Middlessex, England

National Bureau of Standards, Boulder, Colorado

\title{
Cesium Beam Atomic Time and Frequency Standards
}

By

\author{
R. E. BeEhLER, R. C. Mockler, and J. M. Richardson
}

(Received February 23, 1965)

With 10 Figures in the Text

\begin{abstract}
In recognition of the October 1964 declaration of the International Committee of Weights and Measures that the physical measurement of time be based on a particular transition between two hyperfine levels in the ground state of cesium 133, a review of the characteristics of cesium beam atomic frequency standards is presented. This article discusses the general requirements for frequency and time standards, advantages offered by the atomic standard as compared to astronomical standards, various other atomic standards in brief, the operating principles of cesium standards, measures of performance, error sources in cesium standards, characteristics of several standards in current operation, comparison of cesium standards, and atomic time standards derived from atomic frequency standards.
\end{abstract}

\section{Introduction}

The Twelfth General Conference of Weights and Measures, in October 1964, authorized the International Committee of Weights and Measures to designate an atomic or molecular frequency to be used temporarily for the physical measurement of time. The International Commitee declared that the transition to be used is that between the hyperfine levels $F=4$, $m_{F}=0$ and $F=3, m_{F}=0$ of the ground state ${ }^{2} S_{1 / 2}$ of the atom of cesium 133, unperturbed by external fields, and that the value $9,192,631,770 \mathrm{~Hz}$ is assigned to the frequency of this transition.

A review of the characteristics of cesium beam atomic frequency standards is therefore appropriate. This article discusses the general requirements for frequency and time standards, advantages offered by the atomic standard as compared to astronomical standards, various other atomic standards in brief, the operating principles of cesium standards, measures of performance, error sources in cesium standards, characteristics of several standards in current operation, the comparison of cesium standards, and atomic time standards derived from atomic frequency standards.

\section{Requirements of Time Standards}

The unit of the quantity time is the second. Before this unit can be useful in measurement, we must give a definition of it in order to specify its magnitude. The definition is an abstraction. It specifies the idealized concept which underlies the operational realization of the unit. It remains to construct and operate the actual physical apparatus which makes the idealized concept observable. At this point practical variations from ideality occur. We must then distinguish between the ideal definition and the practical apparatus which physically embodies the definition. The qualities of the apparatus are the subject of greatest study, and in our discussion we shall elect to call the apparatus the "standard". The term standard, then, will imply an apparatus based on a particular idealized concept, namely the definition.

It follows that the value provided by the standard may approach the value intended by definition to a greater or lesser degree. The degree to which any prescribed observation of the standard approaches the definition may be termed the accuracy of the standard with respect to the definition, or simply accuracy for short.

A standard of time is taken as a device which generates an ordered, nearly continuous sequence of states, or phases, which can be quantitatively identified and correlated by observation with events - in short, a clock. In practice, clocks are often based on phenomena which recur with almost uniform period. The unit of time will then be proportional to the period of the clock upon which its definition is based.

Since time is a basic quantity in the International System of Units, its standard should satisfy certain requirements deemed desirable for a standard of any quantity.

Firstly, it must have continuity of operation, because of the impossibility of "storing" the unit of time as one "stores", say, the unit of mass. Only if the unit is continuously generated and accumulated into its multiples is it possible to measure an arbitrary interval whenever desired. The only acceptable alternative to continuity is the ability to re-establish the unit and its multiples whenever and wherever needed.

Secondly, as time progresses, the standard must generate a unit which retains constant size with respect to other acceptable measures of time. Of course, a determinable and predictable variability in 
the period of the standard is permissible if corrections may be applied which lead to a constant unit.

Thirdly, accuracy of the standard should equal or excel that of standards based on other possible definitions.

Fourthly, the standard should be accessible to all who need it. If it is not directly accessible, its properties must be made available by indirect means such as calibration or broadcast.

Fifthly, the characteristic period of the standard should be convenient with respect to the operations which are to be performed on it. Time standards give an observable phenomenon $f$ (for example, angular position or voltage) nearly of the form $f(t)=f$ $(t+2 \pi n / \omega)$ where $t$ is the time, $\omega$ is the nominal angular frequency of the standard, and $n$ is an integer. Necessary operations are averaging the period over large values of $n$ for precision, taking Fourier transforms for large $n$ for analysis of the standard, and generating multiples and submultiples of the period for measurement. It is convenient that $\omega$ be large so that these operations can be applied to conveniently small intervals as judged by a time characteristic of man's work, such as his lifetime or the time in which significant changes in techniques occur.

Finally, because of the need to locate events on a continuous scale of time running from some arbitrary origin and common to all observers, the standard should also be capable of continuously accumulating the units. This process will give what is often called epoch. Epoch means the state, or phase, of the standard expressed in the measure of time, as referred to some arbitrary initial state.

\section{Astronomical and Atomic Time Standards}

Up to now, time standards based upon the observed positions of celestial bodies undergoing known or assumed motions have best satisfied the requirements discussed above. Two of the many possible celestial "clocks" have major importance. These are the rotation of the earth as manifested by the apparent diurnal motion of the stars, and the orbital motion of the earth, as manifested by the apparent orbital motion of the sun. The apparent diurnal motion of the sun, which also serves as an important clock, is a combination of these phenomena.

The requirement of continuity of operation over the whole interest span of mankind, and beyond, can hardly be better satisfied than by these celestial standards.

Successive refinements in astronomical knowledge and techniques over the centuries have resulted in closer and closer realization of a unit nearly constant in time. One outstanding improvement was the replacement of the mean solar second by the ephemeris second as the accepted unit. The mean solar second, both in concept and in practice, is subject to fluctuations of the order of 1 part in $10^{8}$ by comparison with other acceptable standards. The ephemeris second is constant in concept, and although there is no evidence that the practical realization of it is other than constant, unpredictable changes and aging effects could occur.

Astronomical standards have always satisfied the requirement of accuracy very well. The standard for the ephemeris second is the observed motion of the moon taken together with its theoretical relationship to the observed motion of the sun. The accuracy of this standard with respect to the definition is a few parts in $10^{9}$.

The requirement of accessibility at any place on earth and at any time is well satisfied by celestial standards. As refinements have been made, however, it has been necessary to rely on a few well equipped observatories to make and reduce the observations and to disseminate the results by appropriate means.

The characteristic period of the celestial standard, presently the tropical year, is inconveniently long. Observations over a few years are necessary to attain precision comparable to the other factors which limit accuracy. Observations over centuries are necessary for an exhaustive understanding of the standard. Interpolation by auxilliary clocks is necessary between observations of the celestial standards.

Astronomical standards provide epoch extremely well. Their continuous operation precludes any lapse which would destroy the relationship of the present epoch to the initial epoch. Their long characteristic periods help avoid ambiguity of phase, even with infrequent observation.

Atomic frequency standards are based upon the frequency $v$ corresponding to a transition between two atomic states separated in energy by $\Delta E$, according to the Bohr relation

$$
h v=\Delta E,
$$

where his Planck's constant. Atomic standards have been developed in the past decade to the point that they satisfy the requirements of good time standards to a degree competitive with astronomical standards.

Continuity of operation has been achieved for intervals of several years. In case of failure, it is possible to re-establish the unit. of time with confidence.

Constancy in the size of the unit based on an atomic definition may be strongly presumed to hold by the nature of atomic energy levels. They are subject to the laws of quantum mechanics and electrodynamics, which enjoy a validity and permanence equal to that of the laws of dynamics underlying the astronomical standards. In particular the levels are determined by the interactions of relatively few elementary particles, and are often amenable to detailed theoretical analysis.

The greatest improvement of atomic standards over astronomical standards has been in the accuracy of the standard with respect to the definition. Accuracy of about 1 part in $10^{11}$ is now typical, and further improvement appears readily possible.

Atomic standards are widely accessible either by construction or purchase. They represent a modest investment in apparatus compared to that needed for astronomical time determinations. Wide radio dissemination of the output of a small number of good atomic standards still remains economically desirable, just as for astronomical standards.

The short characteristic period of the atomic standard is an advantage. Averages over the many cycles necessary to attain statistical precision of measurement are possible in minutes, hours, or days. For example, measurement precision of parts in $10^{13}$ are attainable for averaging times of 12 hours. For this reason, atomic standards make their results 
available more promptly than astronomical standards. Small time intervals down to the nanosecond region are available directly from the standard because of its high frequency without recourse to a separately calibrated oscillator. Present engineering technology has enabled electronic integration of the period of the standard to provide large time intervals of typically several years with negligible error.

Epoch for an atomic time standard may be obtained by such electronic integration of periods. It is a matter of engineering to attain adequate reliability and redundancy. In case of failure of the period integrating apparatus, a lapse in the resulting time scale will occur. This lapse may be bridged by auxiliary standards, such as astronomical standards, but with some loss of accuracy in relating past epochs to present epochs. This loss of accuracy may be quite acceptable however, for many applications.

Atomic standards thus offer equivalent or improved characteristics over astronomical standards for time interval. Both the standards application and technological applications outside the scope of this paper call for the designation of an atomic unit of time interval and a time scale derivable from it. Other units of time interval and other time scales remain important for specialized applications. The ability to transform from one system to another is all that is needed to realize the advantages of all systems.

\section{Various Atomic Frequency Standards}

The cesium standard should be placed in perspective with respect to the various atomic frequency standards. Only in the years subsequent to $1945 \mathrm{did}$ it appear technically feasible to try what had been recognized as possible in principle for some time - to control the rate of a clock by a frequency characteristic of an atom or molecule. I. I. RABI in his 1945 Richtmeyer Lecture before the American Physical Society made the specific suggestion, according to HERSHBERGER and Norton (1948). The frequencies which appeared most suitable were either those characteristic of atomic hyperfine structure or molecular motions such as inversion or rotation. The techniques of observation which developed were absorption at resonance by the gas, the atomic beam method, the maser principle and the optical-microwave double resonance technique.

$\mathrm{NH}_{3}$ Absorption. HERSHBERGER and Norton (1948) gave early results using microwave absorption of the $(J=3, K=3)$ inversion line of $\mathrm{NH}_{3}$ at 23,870 MHz. Basically the technique uses a microwave source, an absorption cell under low pressure to permit well resolved spectral lines, and a detector arranged to display absorption $v s$ frequency. A practical $\mathbf{N H}_{3}$ device (LyoNs, 1952) gave precision of about 2 parts in $10^{8}$ over a run of 8 days. The gas absorption technique is limited mainly by the wide spectral lines, typically $100 \mathrm{kHz}$, produced by Doppler and collision broadening. For this reason, the method is now obsolete for highly precise frequency standards.

Common Features. The remaining techniques of observation all have essential general features in common. Atoms are prepared in a pure state, the state is arranged to have a long lifetime against deexcitation, the atoms are stimulated by microwave radiation to emit (or absorb) in a way free of first-order Doppler effect, and the change of state is detected by convenient means.

Cesium Beam. Atomic beam techniques as described below have been successfully used for the magnetic hyperfine transitions of $C^{133}$ at $9,192 \mathrm{MHz}$. Essen and Parry (1957) at the National Physical Laboratory in England first reported complete success in using the $C s$ resonance to rate an oscillator on a routine basis. Since then, the development of cesium beam standards has been intensive. Several other national standards laboratories have reported results including Canada, United States, and Switzerland (see references).

National standards laboratories in four or five other countries are constructing instruments. Accuracy has been refined to about 1 part in $10^{11}$ as discussed below. The list of references is reasonably complete for cesium standards and reflects the extent of the development.

Thallium Beam. Successful thallium frequency standards have been reported (BoNANom,, 1962; BeEHLER and GLAzE, 1963). The transition used is between the two magnetic hyperfine levels in the ground state, ${ }^{2} P_{1 / 2}$, arising from the coupling of the nuclear spin angular momentum and the electronic angular momentum. The transition is designated as

$$
F=0, m_{F}=0 \rightleftarrows F=1, m_{F}=0
$$

The frequency is $21,310,833,945.9 \pm 0.2 \mathrm{~Hz}$ (BEEHLER and GLAzE, 1965). In principle thallium offers advantages over cesium in a lesser dependence of transition frequency on magnetic field, greater simplicity of the Zeeman spectrum, greater beam signal intensity (since a greater fraction of all atoms in the ground state is in the $m_{F}=0$ states used for the standard frequency transition), and higher frequency than cesium by a factor of two so that a given absolute uncertainty in frequency measurement yields correspondingly greater relative precision. Increased experimental difficulties arise from greater difficulty of deflection and detection. The major limitation on accuracy appears to be phase shift of the $r f$ radiation between the two separated field regions, as discussed below for cesium. Accuracy attained so far is comparable with the accuracy attained for cesium.

$\mathrm{NH}_{3}$ Maser. The first maser used a beam of $\mathrm{NH}_{3}$ molecules prepared in the upper inversion state of the $J=3, K=\mathbf{3}$ rotational state by a quadrupolar electrostatic focuser, which focuses molecules in the upper state and defocuses those in the lower state. (Gordon, Zetger, and Townes, 1954; Basov and Prokнorov, 1955). The beam traverses a microwave resonator, in which the molecules are stimulated to emit by the existing microwave field. In the steady state the radiation emitted maintains a sitored radiation field to stimulate further emission and supplies internal losses of the resonator and external loads. Many subsequent refinements have been developed. Nevertheless, the accuracy of the $\mathrm{NH}_{3}$ beam maser has not proved as great as the accuracy of the cesium beam and the hydrogen maser; yet to be discussed. The reasons concern a complicated dependence of frequency on experimental conditions, partly associated with the unresolved structure of the inversion resonance line. 
Hydrogen Maser. The hydrogen maser (GoLdEx. BERG, KLEPPNER, and RAMSEY, 1960) uses the transi. tion

$$
F=1, m_{F^{\prime}}=0 \rightarrow F=0, m_{F}=0,
$$

between the magnetic hyperfine levels in the ground state, ${ }^{2} S_{1 / 2}$, of atomic hydrogen. The frequency is $1,420,405,751.80 \pm 0.03 \mathrm{~Hz}$ (CRAMPTON, KLEPPNER, and RAMSEY, 1963). The $F=1, m_{F}=0$ state is prepared by magnetic focusing of a beam of atoms, as in atomic beam techniques. A long lifetime in the excited state of 1 to 3 seconds is attained not by time of flight through free space as in the beam maser, but by storage in a bulb. The bulb has walls suitably coated so that wall collisions have a low probability of perturbing the prepared state. The bulb is contained in an electromagnetic resonator, which provides stimulation of radiation and power to external loads just as in the beam maser. First order Doppler shifts are effectively cancelled because of the random directions of the atoms. The technique has several advantages described in the literature (KLEPPNER, GOLDENBERG and RAMSEY, 1962). Stability of the radiation is reported to be about 3 parts in $10^{13}$ over a few days (VESsot and Peters, 1962). Accuracy is estimated at about 1 part in $10^{11}$. Promise of further improvement is good (RAMSEY, 1965).

Optical-Microwave Double Resonance. The optical microwave double resonance technique, often designated as the optically pumped gas cell, emerged about 1957 (KASTLER, 1957). In $R b^{87}$, for example, atoms are preferentially pumped out of the lower $(F=1)$ of two magnetic hyperfine levels by optical resonance radiation. Repopulation of this state by stimulated microwave emission from the $F=2 \rightarrow F=1$ transition is detected at microwave resonance by increased optical absorption of the pumping radiation. Lifetimes in the $F=2$ state are prolonged by an inert buffer gas, collisions with which do not de-excite the $F^{\prime}=2$ state. Inert wall coatings may be used in addition. The buffer gas also performs the essential function of reducing the Doppler width of the radiation. It confines the radiating atom to a region small compared with the wavelength. Thus the motion of the source does not cumulatively affect the phase of the emitted wave, as it does ordinarily in producing the Doppler effect. (Dicke, 1953; WrTTkE and Dicke, 1956). Microwave-optical double resonance devices are convenient and provide short-term stability from day to day of about 1 part in $10^{11}$. Accuracy with respect to the transition frequency of the unperturbed atom suffers from frequency shifts which are not adequately understood, and must be taken as perhaps 1 part in $10^{10}$. These are associated with the buffer gas collisions and with the spectral distribution and intensity of the pumping radiation. Thus the technique is no longer considered suitable for the most accurate frequency standards.

\section{Principles of Cesium Beam Operation}

Hyperfine Structure. The hyperfine splitting in cesium arises because of the interaction between the magnetic moment of the nucleus and the magnetic field produced by the valence electron at the position of the nucleus.

In general, the valence electron produces a magnetic field at the nucleus by virtue of its orbital motion about the nucleus and also by virtue of its intrinsic magnetic moment. These two contributions to the field will be designated by
$\vec{H}_{\text {orbit and }} \vec{H}_{\text {spin }}$ and their sum by $\vec{H}_{\text {el }}=\vec{H}_{\text {orbit }}+\vec{H}_{\text {spin. }}$. The Hamiltonian for the interaction can be concisely written as

$$
\mathscr{H}=-\vec{\mu}_{I} \cdot \vec{H}_{e I},
$$

where $\overrightarrow{\mu_{I}}$ is the magnetic moment of the nucleus. For an alkali atom such as cesium, the ground electronic state is an $S$.state and the orbital angular momentum is therefore zero. Since $\vec{H}_{\text {orbt }}$ is proportional to this angular momentum, the important contribution to $\vec{H}_{\mathrm{el}}$ is $\vec{H}_{\text {spin. }}$. The electron spin has two possible orientations, and we expect two different energy levels for the atom. The ground state for zero external field is thus split by the interaction into two levels and the separation of the two levels is referred to as the hyperfine structure (hfs) separation. If the appropriate quantum formalism is used, the separation $W$ of the two levels is given approximately by (Kopfermann, 1948)

$$
\begin{aligned}
W & =\left(I J F m_{F}\left|-\overrightarrow{\mu_{I}} \cdot \vec{H}_{e l}\right| I J F m_{F}\right) \\
& =\frac{g_{I} \mu_{N} \mu_{0} Z^{3}}{a_{0}^{8} n^{3}}\left[\frac{F(F+1)-I(I+1)-J(J+1)}{J(J+1)\left(L+\frac{1}{2}\right)}\right],
\end{aligned}
$$

where $g_{I}$ is the nuclear $g$-factor, $\mu_{N}$ is the nuclear magneton, $\mu_{0}$ is the Bohr magneton, $Z$ is the atomic number, $a_{0}$ is the radius of the first Bohr orbit $\left(a_{0}=\hbar^{2} / m e^{2}\right), n$ is the principle quantum number, $F$ is the total angular momentum quantum

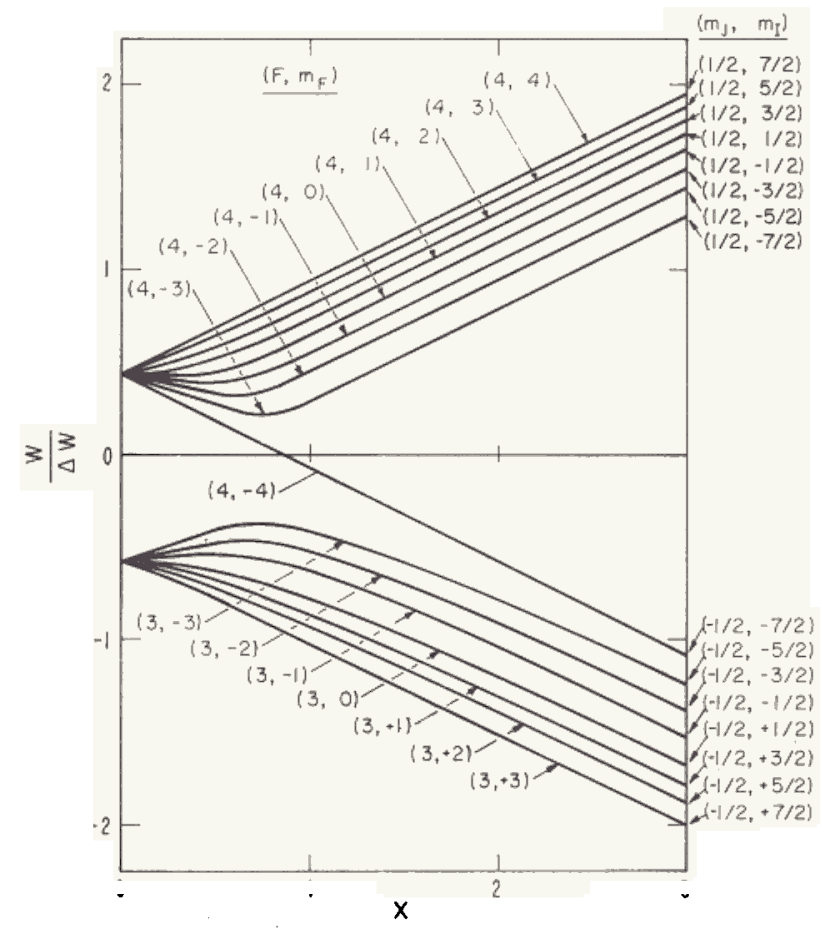
Fig. 1. Energy level diagram of $C_{\delta}{ }^{13 s}$ in the ${ }^{2} S_{1 / 2}$ ground state as a function
of the applied magnetic fleld

number, $I$ is the nuclear angular momentum quantum number, $J$ is the total electronic angular momentum quantum number $\overrightarrow{(J}=\vec{L}+\vec{S}), L$ is the orbital angular momentum quantum number for the valence electron, and $m_{F}$ is the magnetic quantum number associated with $F$. The level separation was first worked out by FERMI (1930) and FERMI and SEGRE (1933). Equation (3) reduces to their result for $J=1 / 2$ and $L=0$.

Effect of External Field. If an external field is applied, certain degeneracies are removed as shown in Fig. 1. This energy level diagram is for the ground state of cesium for which $I=7 / 2$, and $J=1 / 2$. In this case, since $F=I+J$, $I+J-1, \ldots, I-J$, there are only two $F$ levels: $F=I \pm 1 / 2$ or $F=4$ and $F=3$. Their energy as a function of the applied field $H$ is given by the Breit-Rabi formula (RAMSEY, 1956):

$$
\begin{gathered}
W_{F=} I \pm \frac{1}{2}, m_{F}=\frac{-\Delta W}{2(2 I+1)}+\mu_{o} g_{I} m_{F} H \pm \\
\frac{\Delta W}{2}\left[1+\frac{4 m_{P} x}{(2 I+1)}+x^{2}\right]^{\frac{1}{2}},
\end{gathered}
$$


where $\Delta W$ is the hfs separation, given approximately by the Fermi-Segre formula, between the $F=I+1 / 2$ and $F=I-1 / 2$ states in zero field; and

$$
x=\frac{\left(g_{J}-g_{1}\right) \mu_{o} H}{\Delta W} .
$$

For a small applied field $\vec{H}$ the energy levels may be drawn as in Fig. 2. From Fig. 1 and 2 it is evident that the states $\left(F=4, m_{F}=0\right)$ and $\left(F=3, m_{F}=0\right)$ are the least sensitive to
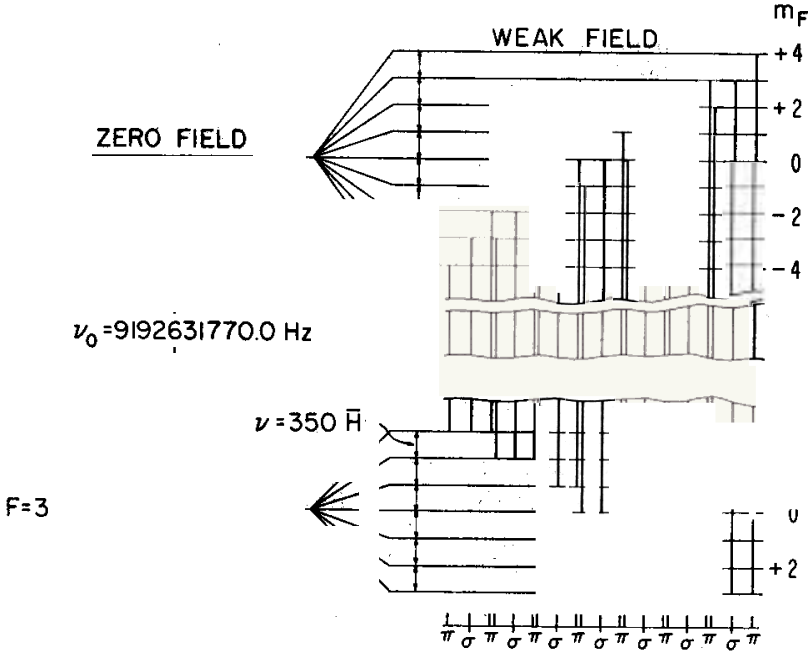

Fig. 2. Energy level diagram for $C s^{133}$ in a weak applied magnetic fleld

the applied field. Since the accuracy with which we can measure the transition frequency is partly determined by how accurately we can measure $H$, we choose this field insensitive transition for our frequency standard. The frequency is slightly field sensitive, and one can show from the Breit-Rabi formula that the frequency is given by

$$
\nu=\nu_{0}+427 H^{2}
$$

for small values of $H$, where $\nu$ is the frequency in $\mathrm{Hz}$ of the $\left(F=4, m_{F}=0\right) \longleftrightarrow\left(F=3, m_{F}=0\right)$ transition when the applied

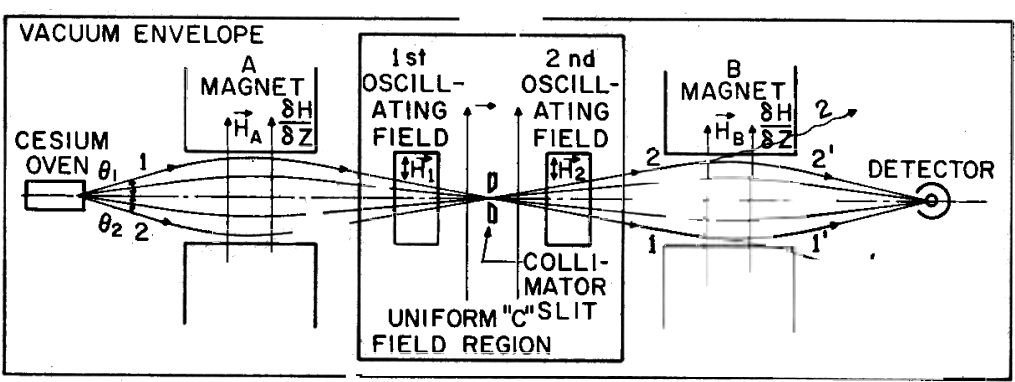

Fig. 3. A schematic of a typical atomic beam spectrometer
The effective magnetic moment has, in general, a different value for each state:

$$
\mu_{\mathrm{eft}}=\mu_{F}=I \pm \frac{1}{2}, m_{F} \quad g_{I} \mu_{0} m_{F}\left\{\frac{\frac{x}{2}+\frac{m_{F}}{(2 I+1)}}{\left[1+\frac{4 m_{F} x}{(2 I+1)}+x^{2}\right]^{\frac{1}{2}}}\right\} .
$$

Evidently, then, the force on the atom depends not only on the gradient of the field but also upon the particular state that the individual atom is in. The effective magnetic moment is positive for some states and negative for others. The magnet is designed so that the gradient of the field has a direction transverse to the beam and in the plane of the drawing of Fig. 3.

The quantity $\mu_{\mathrm{erz}} / \mu_{0}$ is plotted as a function of the applied field $H$ in Fig. 4 . It is simply given by the negative derivatives of the curves plotted in Fig. 1. Suppose the positive $z$ direction is in the upward direction perpendicular to the spectrometer axis in Fig. 4 . Let $\overrightarrow{e_{z}}$ be a unit vector in the $z$ direction. The magnets are designed so that

$$
\vec{F}=\mu_{\mathrm{eft}} \nabla H=\vec{e}_{2} \mu_{\mathrm{ett}} \frac{\partial H}{n}
$$

and if $\mu_{\text {eft }}$ is negative, as it is for the $(4,0)$ state (i. e., the $F=4, m_{F}=0$ state, see Fig. 4), then the force is downward. If $\mu_{\text {etr }}$ is positive, as it is for the $(3,0)$ state, the force is upward in the figure. Atoms effusing from the oven in the $\theta_{1}$ direction along trajectory 1 (see Fig. 3 ) that are in the $(4,0)$ state will experience a downward force. Some of them, those with the proper velocity, pass through the collimator slit and pass on to the second deflecting field region. Those atoms in state $(3,0)$ effusing in the $\theta_{1}$ direction experience an upward force and do not pass through the collimating slit. They are eliminated from the beam. The opposite is true for atoms effusing in the $\theta_{2}$ direction. Those in state $(3,0)$, experiencing an upward force pass through the collimator, and those in the $(4,0)$ state are eliminated from the beam and pass into the second deflecting field region. Normally the deflecting fields are high fields so that atoms in states 4,$4 ; 4,3 ; 4,2 ; 4,1 ; 4,0 ; 4,-1$; $4,-2$; and $4,-3$ effusing at a positive angle $\theta_{1}$ pass through the collimator. Correspondingly, atoms in states $4,-4 ; 3,3$; 3,$2 ; 3,1 ; 3,0 ; 3,-1 ; 3,-2 ;$ and $3,-3$ effusing at a negative angle $\theta_{2}$ pass through the collimator.

If the second deflecting field (that produced by the $B$ magnet) is identical to the $A$-field, the atoms in, for example, the $(4,0)$ state following trajectory 1 (see Fig. 3) experience a downward force as in the $A$-field. They will not strike the detector. Similarly, atoms in the $(3,0)$ state, say, following trajectory 2 experience an upward force. They too will miss the detector. Suppose now that a radiation field is applied in the uniform field region (the $C$-field region, applied in order to preserve the state identy of an atom passing from the $A$-field to the $B$-field) of frequency appropriate to the $(4,0) \longleftrightarrow(3,0)$ transition. Atoms in the $(4,0)$ state are induced to emit a quantum of energy, and atoms in the $(3,0)$ field has a magnitude of $H$ (in oersteds). $v_{0}$ is the transition frequency for $H=0$; it has the value $9192631770.00 \ldots \mathrm{Hz}$.

Method of Observation. Now let us see how we observe these transitions with the atomic beam technique. The atomic beam spectrometer has the basic structure shown in Fig. 3 . Neutral atoms effuse from the oven and pass through the nonuniform magnetic field of the $A$ deflecting magnet. The atoms have a magnetic dipole moment $\mu$ and consequently experience a transverse force in this $A$-field region. This force is given by

$$
F=-\nabla W=-\frac{\partial W}{\partial H} \nabla H,
$$

where $W$ is the potential energy of a dipole in the field and depends on $H$ only according to the Breit-Rabi formula (4). The effective magnetic moment is

$$
\mu_{\mathrm{efr}}=-\frac{\partial W}{\partial H},
$$

by analogy with the case where the dipole moment is independent of $H$. Hence

$$
\vec{F}=\mu_{\mathrm{eft}} \nabla \boldsymbol{H}
$$
state are induced to absorb a quantum of energy. Atoms that were in the $(4,0)$ state following a trajectory 1 now find themselves in the $(3,0)$ state. Atoms that were in the $(3,0)$ states following a trajectory 2 now find themselves in the $(4,0)$ state. Each sef atom that has made the transition has had its mag. netic moments "flipped". As a result of this change in sign of the magnetic moment, the forces on these atoms in the $B$ deflecting region will also change sign. These atoms will now follow the trajectories $1^{\prime}$ and $2^{\prime}$ in Fig. 3 and strike the detector.

By means of the well-known surface-ionization process (ZANDBERG and Ionov, 1959; Datz and TAYLOR, 1956) the atoms are converted into positively-charged ions with nearly $100 \%$ efficiency and can then be collected and measured. The resulting detected beam current goes through a maximum when the frequency of the radiation field is swept through the resonant frequency of the cesium transition. An actual recording of the spectral line shape, obtained in the process, is shown in Fig. 5 for the usual case of two separated excitation regions (Ramsey technique). The appearance of the auxilliary peaks on either side of the central resonance peak is a consequence of the separated oscillating-field technique. (RAMSEY, 1950; RAMSEY, 1956). The advantages offered by the Ramsey method 
include a narrower width of the spectral line and less severe requirements on the uniformity of the $C$-field as compared to the single excitation region case (Rabi technique).

Unlike many other resonance experiments, the transition probability is examined by observing the effect of the radiation on the beam rather than the effect of the beam on the radiation field. The intensity of the signal does not depend upon the population difference of the two states but on the sum of their populations. The spectral linewidth is given by the uncertainty relation $\Delta E \Delta t \approx h$ or $\Delta v \Delta t \approx l$, where $\Delta v$ is the

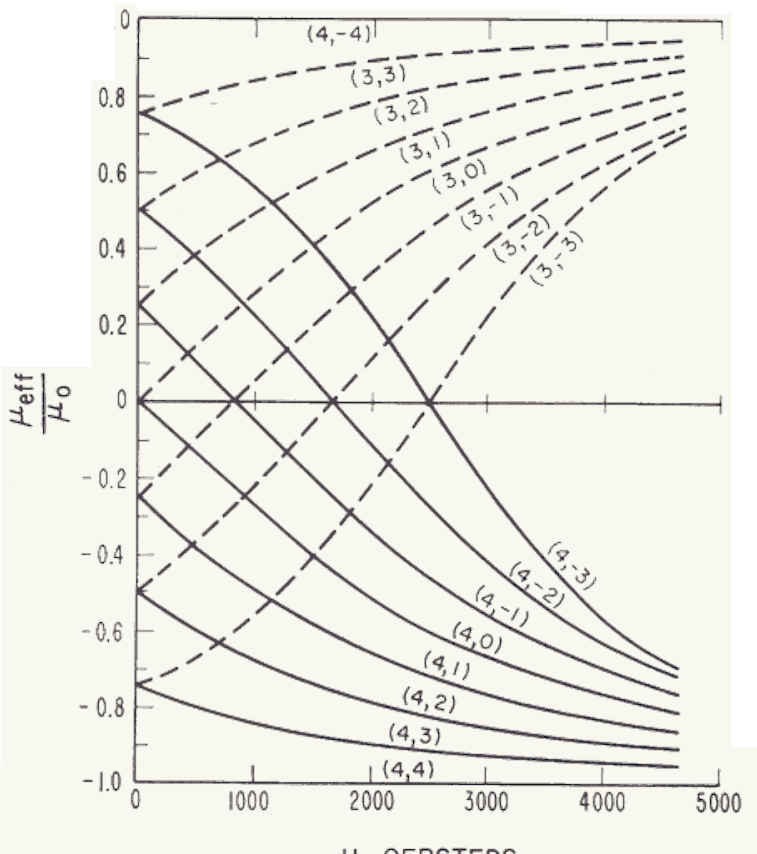

$\mathrm{H}$, OERSTEDS

Fig. 4. The effective magnetic moment relative to the Bohr magneton for the various magnetic substates in $\mathrm{Cs}^{133}$ as a function of the applied magneti fielo

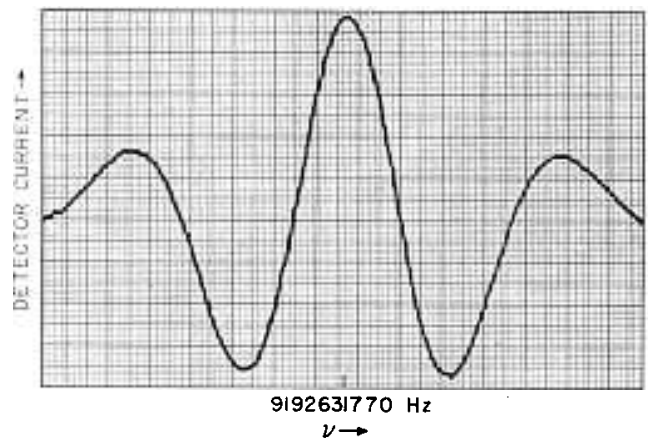

Fig. 5. Recording of the central portion of the Ramsey resonance line shape for one of the National Bureau of Standard's cesium standards

linewidth and $\Delta t$ is the average transit time of an atom through the radiation field, if a single radiation field is used. If two radiation fields are used to induce the transition, then $\Delta t$ is the average transit time for an atom to go from the first field to the second. The linewidth thus depends on the average velocity $v$ in the beam and the length of the transition region, since $\Delta t=L / v$.

The process of measuring the frequency of an unknown source in terms of the cesium standard usually involves the direct comparison of the unknown frequency with the known frequency of an oscillator which is controlled in some manner by the atomic resonance. In the most common technique employed the control is done automatically and continuously by using an electronic servo system. The controlled oscillator frequency - normally near $5 \mathrm{MHz}$ - is modulated at a low audio rate and then multiplied to the cesium frequency at $9192 \mathrm{MHz}$. If the multiplied frequency differs even slightly from that of the resonance maximum, the modulation results in the generation of a component of the detector current at the modulation frequency. If this component is amplified and phase-compared with the original modulation signal, it can be shown that the $D C$ output signal of the phase detector has a magnitude proportional to the amount of deviation of the applied frequency from the atomic resonance frequency and a sign which depends on whether the applied frequency is higher or lower than the resonance. Thus, this signal may be used with appropriate circuitry to correct automatically and continuously the frequency of the $5 \mathrm{MHz}$ oscillator to a value which bears a fixed and known relationship to the defined cesium frequency.

In a somewhat different technique used with some laboratory-type standards the control of the $5 \mathrm{MHz}$ oscillator is accomplished manually. In this case an operator manually adjusts this frequency to a value which results in a maximum current from the detector corresponding to a maximum proba. bility for the atomic transition.

\section{Measures of Periormance}

There are no universally accepted measures for describing the performance of atomic frequency standards. Until common agreement on these measures is reached, each author is obliged to state exactly how his estimates of performance are to be interpreted.

Fluctuations may occur (a) among various observations for a given adjustment of a given instrument, (b) among various independent adjustments of a given instrument, and (c) among various independent instruments. Usually these fluctuations increase in magnitude in the order mentioned. Each of these types of fluctuations gives useful information about the performance of the standards. The fluctuations of type (a) arise from fluctuations of instrumental and environmental parameters during operation. The fluctuations of type (b) contain fluctuations of type (a) and also fluctuations in setting the adjustable parameters from time to time. The fluctuations of type (c) contain types (a) and (b) and also fluctuations from instrument to instrument in fixing the non-adjustable parameters.

Stability. A sequence of $n$ readings of a particular standard in a particular adjustment, against a comparison oscillator assumed temporarily constant, will show fluctuations. The standard deviation of these observations is often called the stability of the standard. Tts value will depend on the duration of the individual observations, and many be qualified as short term or long term stability. For example, if the average frequency over 1 second is measured for $n=100$ successive times, the standard deviation of such a 1 -second observation as estimated from the sample of 100 observations may be called the "short term stability" applicable to 1 second duration. In this sense, stability indicates the degree to which the standard in steady operation gives results constant in time.

Of interest is not only the magnitude of the frequency fluctuations but also their rate. Thus a more complete characterization of the stability of a given standard in a given state of adjustment would be all the statistical properties of the frequency fluctuations $F(t)$ about a mean frequency $\bar{\nu}$ considered as a function of time, that is $F(t)=v(t)-\bar{v}$ where $v(t)$ is the instantaneous frequency. These would be the moments of the amplitude distribution of $F(t)$, its spectrum, its autocorrelation function, and so forth. Studies of such properties are in progress but are beyond the scope of this paper.

Precision. Precision is considered a property of a measurement process, not of an instrument alone. It measures the random error in carrying out a prescribed observation. For example, if the prescribed observation is the mean of $n$ successive readings taken as described in the preceding section, the sample standard deviation of the mean would appropriately measure the precision of such an observation.

Reproducibility. A sequence of comparisons for indepenent adjustments of a particular standard, against a reference standard assumed available and temporarily constant, will yield a mean and a standard deviation. The standard deviation of such observations may be called the reproducibility of the instrument. In this sense reproducibility indicates the degree of resettability of the instrument.

Accuracy. We use accuracy in this paper as the degree to which any prescribed observation of a particular standard approaches the definition. It requires (a) an estimate of the random error in carrying out the prescribed observation for a given adjustment (that is, precision), (b) an estimate of systematic errors incurred in adjusting the standard (that is, reproducibility), and (c) an estimate of systematic errors 
incurred in constructing the standard. The measure of all these errors should be stated; that is, whether they represent limits of error, or one-, two-, or three-sigma estimates of a distribution of values. The method of combination should be stated. If the errors may be considered independent, for example, the square root of the sum of their squares may be taken as a proper estimate of the total error.

The estimate of precision of an observation has been discussed above.

We must now distinguish between possible ways of making the last two estimates, (b) and (c) depending on whether the standard to be evaluated stands in the highest echelon so that no higher reference standards is available, or whether the standard is in a lower echelon and may be compared with a reference standard. If the standard stands alone, the last two estimates must be made by a study of the effect of all known parameters on the frequency and an estimate of the uncertainty in these parameters in a given instrument or for a given adjustment.

A somewhat different viewpoint is possible if there are several standards of comparable quality in the highest echelon. We may then agree to consider all these as a sample from an ensemble of standards. They may be compared among one another with respect to the sample mean. Then the sample standard deviation may be taken as a single overall estimate of the accuracy of any one of the standards. This view assumes that all members of the ensemble randomly partake of variations in the parameters and are stationary over the time required in practice, often lengthy, to effect the comparisons.

If a reference standard is available, then accuracy with respect to the reference standard is often taken simply as the mean difference of successive comparisons for independent adjustments of the test standard. This view assumes that the precision of the comparison may be made arbitrarily small by increasing the number of observations.

Confusion of Terminology. Various authors have used the concepts explained here but under various names. We thus find "precision" used for our concept of "stability", as well as for our concept of "reproducibility". The reader is cautioned against this confusion.

\section{Error Sourees in Cesium Beam Standards}

Systematic errors in cesium beam standards may arise from a great variety of sources. Fortunately, however, the atomic beam magnetic resonance technique used in cesium standard has the virtue that most of these errors can be kept quite small, providing that reasonable care is exercised in the design and construction of the standard. It has been found possible in at least two national standards laboratories to reduce the combined systematic error from all known sources to a level of $\pm 1 \times 10^{-11}$. In the following discussion an attempt will be made to mention at least briefly all the known error sources that may contribute significantly to the inaccuracy of a cesium standard. In some case quantitative results will be given based on the authors' experience with three independent laboratory-type standards at NBS. The error sources will be grouped depending on whether they produce a displacement of the resonance line, an asymmetrical distortion of the resonance line shape, or an erroneous measurement of the resonance peak even though the line is neither displaced nor distorted.

\section{Errors Which Result from a Displacement of the Resonance}

Uncertainty in Magnetic Field. The dependence of the cesium $(4,0) \longleftrightarrow(3,0)$ transition frequency on the magnitude of the uniform magnetic $C$ field is given by

$$
\nu=v_{0}+427 \overline{{H^{2}}^{2}(x)} \text {, }
$$

where $\nu$ and $v_{0}$ are in $\mathrm{Hz}, \mathrm{H}$ is in oersteds, and $\overline{\mathrm{H}^{2}(x)}$ is a spatial average over the length between the two oscillating field regions. Any uncertainties in determing $\overline{\mathbf{H}^{2}(x)}$ will lead to corresponding errors in frequency measurements referred to $\nu_{0}$, the zero-field standard frequency. In practice, the field is determined from frequency measurements of various fieldsensitive transitions in cesium by using theoretical relations between frequency and field magnitude. The most commonly used transitions for this puspose are the low frequency transitions between the various $m_{F}$ levels within a single $F$ state, such as the $(4,-4) \longleftrightarrow$ $(4,-3)$ transition, and certain microwave transitions, such as the $(4,1) \longleftrightarrow(3,1)$ transition (see Fig. 2). The appropriate frequency-field relationships are: and

$$
\nu=3.5 \times 10^{5} \overline{H(x)} \text { for }(4,-4) \leftrightarrow(4,-3)
$$

$$
\nu=v_{0}+7 \times 10^{5} \overline{H(x)} \text { for }(4,1) \leftrightarrow(3,1) .
$$

The value of $\overline{H(x)}$ obtained in this manner is then normally squared and used in equation (9) in place of $\overline{\mathrm{H}^{2}(x)}$, which is much more difficult to determine. Thus, frequency uncertainties resulting from this procedure arise from two sources:

(a) uncertainties in the value of $\overline{\mathrm{H}(x)}$, and

(b) uncertainties due to the use of $\overline{\mathrm{H}(x)}{ }^{2}$ for $\overline{\mathrm{H}^{2}(x)}$.

In order to establish the uncertainty in frequency which results from an uncertainty in $\overline{\mathbf{H}(x)}$, we can give $H(x)$ a constant increment $\Delta H$ and compute the resulting $\Delta v$ from equation (9). Thus,

$$
\begin{aligned}
v & +\Delta v=v_{0}+427 \overline{[H(x)+\Delta H]^{2}} . \\
& \left.=v_{0}+427 \overline{\left[H^{2}(x)\right.}+\overline{2 H(x)} \Delta H+(\Delta H)^{2}\right] .
\end{aligned}
$$

Neglecting the square of the differential and subtracting $\nu$ from both sides, again using (9), gives

$$
\Delta v=2(427) \overline{H(x)} \Delta H .
$$

The procedure followed at NBS for determining the uncertainty in $\overline{H(x)}$ consists of measuring four different independent microwave transitions for each setting of the current which produces the $C$ field. A least-square fit of a straight line is made to these data, and the uncertainty to be associated with any field value obtained from this calibration is taken to be the computed standard deviation of a point from this line. This error contribution amounts to $\pm 1.9 \times 10^{-5}$ oersted or $\pm 1 \times 10^{-13}$ in frequency for the normal field value used of .050 oersted.

Longitudinal Non-uniformity of Magnetic Field. The uncertainty from the second source mentioned arises becauses $\overline{\mathrm{H}^{2}(x)}$, which is the quantity that determines the true value of $\nu$, is not necessarily equal to $\overline{\mathrm{H}(x)}^{2}$, which is the value determined and used in practice. The error in $\mathrm{Hz}$ in using the latter quantity is given by

$$
\left.\Delta v=427 \overline{\left[H^{2}(x)\right.}-\overline{H(x)^{2}}\right] .
$$

It can readily be shown by direct substitution that if the $C$ field is expressed as

$$
\mathrm{H}(x)=\mathbf{H}_{0}(x)+c I,
$$

where $H_{0}(x)$ is the non-uniform residual field in the drift-space region with the current $I$ turned off and $c$ is a constant, then

$$
\Delta v=427\left[\overline{H_{0}^{2}}(x)-\overline{H_{0}(x)}{ }^{2}\right] .
$$


If sufficiently detailed knowledge of the field uniformity can be obtained in some manner, this error can be evaluated and applied as a correction to all frequency measurements. Fig. 6 shows a plot of the residual field uniformity in one of the NBS standards obtained by drawing a very small sensitive magnetometer probe along the drift-space region in the position normally occupied by the atomic beam. From these data $\left.\overline{\left[\mathrm{H}_{0}^{2}(x)\right.}-\overline{\mathrm{H}_{0}(x)}{ }^{2}\right]$ is found to be $0.14 \times 10^{-6}$ oersteds ${ }^{2}$ resulting in a frequency error given by equation (17) of less than $1 \times 10^{-14}$. Since this non-uniformity is measured only infrequently, however, and is known to be somewhat dependent on external conditions which may vary with time, it is considered preferable to treat the computed value $\Delta v$ as an uncertainty rather than a known correction.

Transverse Non-uniformity of Magnetic Field. Nonuniformity of field across the beam cross section may also produce frequency errors. This effect produces a broadening of the field-sensitive microwave resonance line with respect to the field-insensitive $(4,0) \longleftrightarrow(3,0)$

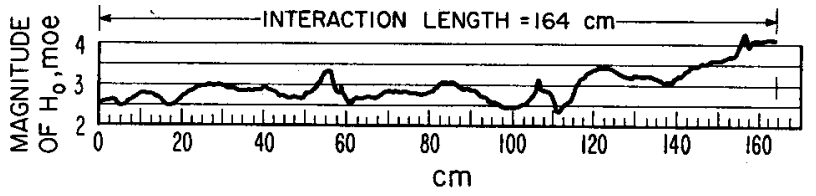

Fig. 6. Plot of the variation of the magnitude of the residual magnetic field $\mathrm{H}_{0}(x)$ along the length of the $C$-field region in NBS II

line, thus offering a means of detecting the existence of this condition.

Doppler Effects. The presence of first and second order Doppler effects will also produce a displacement of the resonance. In general, one does not expect any first order Doppler shifts in this type of standard, since ideally the radiation field in the cavity consists of two equal traveling waves going perpendicular to the velocity of the atoms. If, however, due to imperfect alignment the atoms have a component of their velocity parallel to the radiation field and a net traveling wave exists in the cavity, a frequency shift $\Delta \nu$ may occur given by

$$
\Delta v=\frac{1-R}{R} \nu \frac{\bar{v}}{c} \sin \alpha,
$$

where $R$ is the power reflection coefficient, $v$ is the mean speed of the atoms in the beam, and $\alpha$ is the angle between the waveguide and a line normal to the beam. The experimental verification of such a shift has not as yet been made conclusively.

The unavoidable second order Doppler shift given by

$$
\Delta v=v^{2} / 2 c^{2}
$$

amounts to about $3 \times 10^{-13}$ and is negligible for most present applications.

Electric Fields. Possible frequency shifts due to electric fields are well beyond present measurement precision - at least for fields on the order of a few volts/cm. (HAUN and ZACHARIAS, 1957).

\section{Errors Which Result from a Distortion . of the Resonance Shape}

Longitudinal Non-uniformity of Magnetic Field. If the $C$-field is non-uniform along its length in such a way that its magnitude at the two localized regions of oscillating field is not equal to its average value over the entire drift space, the Ramsey resonance pattern will not be centered on the broad Rabi resonance pedestal. The resultant asymmetrical distortion of the Ramsey line will lead to a frequency error, since the peak of the line is then shifted from its undisturbed value. Observation of the symmetry of the fieldsensitive microwave transitions such as the $(4,1) \longleftrightarrow$ $(3,1)$ provides a very sensitive indication of the longitudinal non-uniformity. The amount of observed asymmetry can be related to the degree of non-uniformity, thus permitting an estimate of the resultant error to be made.

RAMSEY has shown that if the magnitude of the $C$ field varies within the small oscillating field regions large frequency shifts may result. (RAMsEx, 1959). The presence of such a shift may be detected rather easily, since the amount of error depends strongly upon the intensity of the radiation field in the cavity.

Polarity Dependent Shifts. In a number of standards now in operation frequency shifts of up to $1 \times 10^{-10}$ have been observed upon reversing the polarity of the $C$ field. (Bonanomi, 1962; Beehler and Glaze, 1963; Essen, Stemle, and Sutcliffe, 1964). The sources of these shifts have been attributed to either a Millman effect or the influence of a rather large residual field in the transition region. The Millman effect is due to the beam experiencing an oscillating field which changes direction along the beam path. Such a condition may occur if there is leakage of the radiation field in the cavity out the beam entrance and exit holes. Since the direction of the resulting frequency shift changes upon reversal of the $C$-field direction, the error may be eliminated by making frequency measurements for both field polarities and using the mean value.

If a residual static field exists in such a direction as to cause the magnitude of the resultant field to vary when the component due to the $C$-field current is reversed, the measured transition frequency uncorrected to zero field will depend on the polarity. However, in this case the value of $\overline{\mathbf{H}}$ given by the field calibration will also depend on the $C$-field polarity in such a way that no error should result in the measured frequency referred to zero field after the $427 \mathrm{H}^{2}$ correction is applied to the data.

Phase Difference. If a difference in phase exists between the radiation fields at the two ends of the microwave resonant cavity, the Ramsey resonance curve will be distorted asymmetrically with a resulting frequency error. Although in an ideal lossless single microwave resonant cavity there would be no phase difference, it is usually found in practice that unavoidable losses and electrical asymmetry do exist which cause phase shifts. KarTaschoff (KARTASCHOFr, 1962) has shown that the phase shift $\Delta \varphi$ due to an electrical asymmetry $\xi$ in length units in a cavity of length $L$ and attenuation constant per unit length $\alpha$ can be expressed as

$$
\Delta \varphi=\alpha L \beta \xi,
$$

where $\beta$ is the propagation constant of the waveguide. Since the phase difference $\Delta \varphi$ will result in a frequency error $\frac{\Delta v}{\nu}=\frac{\Delta \varphi}{\pi Q}$, where $Q=$ the quality factor of the 
resonance line, the frequency shift can be computed for a given electrical asymmetry. Note that this particular error is independent of beam length. These relations place rather severe mechanioal tolerances on the cavity, since an asymmetry of only $0.12 \mathrm{~mm}$ will produce an error of about $1 \times 10^{-12}$. Experience at NBS has shown that phase shifts sufficient to cause frequency errors of $1 \times 10^{-11}$ may also develop gradually with time as a result of the accumulation of cesium and pump oil deposits within the cavity ends. Periodic cleaning of the cavity structure is necessary in this case.

A means of detecting the presence of cavity phase shifts and correcting for their effect is provided by the fact that the direction of the resulting frequency shift from the true value changes with the direction in which the beam traverses the cavity. Therefore, if the standard is designed to allow for the physical rotation of the cavity structure by $180^{\circ}$ without disassembly of the cavity, or if the direction of beam traversal can be changed by interchanging the oven and detector, one-half of the frequency shift observed as a result of either of these operations can be used as a correction to all data, so that only the uncertainty in determining the phase shift contributes to the inaccuracy of the standard. Both methods have been utilized at NBS with the tentative conclusion that the more reproducible data is obtained with the second technique, possibly due to the unavoidable changing stresses caused by actual rotation of the cavity. The uncertainty in the correction as determined from the reproducibility is $\pm 3 \times 10^{-12}$ for NBS III. Attempts have also been made in some laboratories to detect phase shift errors by a careful examination of the Ramsey line symmetry. Signal-to-noise ratios achieved in present standards, however, limit the minimum detectable asymmetry to amounts corresponding to frequency shifts of $1 \times 10^{-10}$ or greater.

Cavity Mistuning. A second possible error source associated with the microwave cavity is the cavity pulling effect which occurs if the resonance frequency of the cavity is not tuned exactly to the cesium frequency. The magnitude of this shift is given by

where

$$
\Delta v_{L}=\left(Q_{c} / Q_{L}\right)^{2} \Delta v_{c},
$$

$$
\begin{aligned}
& \Delta v_{L}=\text { the frequency error of the Ramsey line } \\
& \Delta v_{c}=\text { the amount by which the cavity is mistuned } \\
& Q_{c}=\text { the } Q \text { of the cavity } \\
& Q_{L}=\text { the } Q \text { of the Ramsey line. }
\end{aligned}
$$

For a cavity $Q$ of 5000 and a line width of $100 \mathrm{~Hz}$ the calculated shift is about $3 \times 10^{-13}$ for a $1 \mathrm{MHz}$ detuning. In practice, the cavity can usually be kept tuned to within much narrower limits than $\pm 1 \mathrm{MHz}$.

Neighboring Transitions. Another possible error contribution which may be quite significant under some conditions arises from distortion of the line by overlap of other microwave transitions in the cesium spectrum. Assuming that the parallelism of the $C$ field and the oscillating fields is maintained properly so that $\pi$ transitions $\left(\Delta m_{F^{\prime}}= \pm 1\right)$ are not excited, the nearest transitions to the $(4,0) \leftrightarrow(3,0)$ are the $(4,1) \longleftrightarrow(3,1)$ and $(4,-1) \longleftrightarrow(3,-1) \sigma$ resonances $\left(\Delta m_{F}=0\right)$ which are located symmetrically above and below the $(4,0) \longleftrightarrow(3,0)$ in frequency. Ideally these neighboring lines are symmetric about the $(4,0)$ $\longleftrightarrow(3,0)$ both in frequency and intensity so that no net overlap shift should occur. However, it is usually found in practice that due to imperfect beam alignment the amplitudes of these two neighboring transitions are not equal. The resulting shift in the $(4,0) \longleftrightarrow$ $(3,0)$ frequency in this case depends on the linewidths of the overlapping lines, the amount of asymmetry existing in the intensities, and especially on the separation in frequency of the overlapping lines from the $(4,0) \longleftrightarrow(3,0)$ (Mockler, BeEHLER, and SNIDER, 1960). This amount of separation depends on the magnitude of the $C$ field according to (11), so that the surest way of avoiding this effect is to operate at a sufficiently high $C$ field - normally, above 0.040 oersteds - so that significant overlap does not occur. In some of the early NBS work on cesium standards at fields of only 0.020 oersteds it was discovered that a systematic error of greater than $4 \times 10^{-11}$ was being introduced into the data from an overlap effect.

Spectral Purity. The spectral purity of the microwave signal used to excite the cesium resonance has been found to be extremely important (BARNes and MockLER, 1960). When the transition is excited with a signal which contains sidebands at a significant level, particularly if the sidebands are asymmetrically distributed about the carrier, large frequency shifts in the $(4,0) \longleftrightarrow(3,0)$ standard frequency may result. For example, excitation of the cesium resonance in the original NBS cesium standard at one time yielded a frequency shifted by $32 \times 10^{-10}$. Examination of the spectrum using a high-resolution ammonia-maser spectrum analyzer system (BARNES and HEIM, 1961) showed the presence of large, asymmetrical sidebands at the power line frequency and its harmonics. If the excitation contains a single unbalanced sideband at a frequency $v_{s}$, the frequency shift produced is (RAMSEY, 1956)

$$
\begin{gathered}
\quad \frac{\Delta v}{v_{0}}=\frac{A_{t}}{A_{0}} \frac{W^{2}}{v_{0}\left(v_{0}-v_{s}\right)}, \\
A_{s}=\text { amplitude of sideband } \\
A_{0}=\text { amplitude of carrier at } v_{0} \\
W=\text { resonance width } \\
v_{0}=\text { cesium resonance frequency. }
\end{gathered}
$$

The advantage of a narrow resonance line is readily apparent. This relation also applies if $A$ is the difference in amplitude of two unbalanced sidebands. Since the sideband intensity is multiplied by the factor of frequency multiplication, those which originate in the driving oscillator (usually near $5 \mathrm{MHz}$ ) or early stages of the multiplier chain are particularly serious. For example, a sideband $100 \mathrm{db}$ below the carrier at the oscillator frequency of $5 \mathrm{MHz}$ will be only $35 \mathrm{db}$ below the carrier at cesium frequency, even if the multiplier chain adds no noise of its own. If the most significant sidebands are due to power line frequencies and its harmonics, as is often the case, the spectrum may change as ground loops and electrical connections are changed in the laboratory resulting in time-varying frequency errors. Ideally, the spectrum of the excitation should be monitored frequently with a highresolution spectrum analyzer. As a practical compromise at NBS, the beat frequency at approximately $100 \mathrm{~Hz}$ between the normal cesium excitation signal and a similar signal from another oscillator-multiplier 
chain system which is known to have a clean spectrum is monitored regularly on an oscilloscope. The presence of noise, modulations, or other deviations from a sinusoid are watched for. This simple system is sufficiently sensitive that on several occasions undesirable modulations have been detected which were causing frequency errors of less than $2 \times 10^{-11}$.

\section{Errors Which are Present Even When the Resonance is Perfect}

Some contribution to inaccuracy arise because of errors involved in the measurement of the resonance peak, even though the line itself is undistorted and not displaced.

Random Fluctuations. Fig. 7 shows data from a 48-hour continuous comparison of two independent cesium standards, designated NBS II and NBS III, maintained at the National Bureaú of Standards (NBS) in Boulder, Colorado. Each plotted point, representing the difference frequency averaged over 1 hour, is the mean of twenty 3-minute measurements of the period of the beat frequency. The length of the vertical bar drawn at each point represents the computed standard error of the 1-hour observation (standard deviation of the mean of the twenty 3-minute averages) and is thus an estimate of the relative precision of this process. This precision figure is typically $\pm 7 \times 10^{-13}$ for this data.

A second estimate of the frequency fluctuations appropriate for 1 hour averaging times can be obtained from these data by computing the standard deviation of the 481 -hour averages. This value is $\pm 1 \times 10^{-12}$. It can easily be shown that if the measurement process is in statistical control - i. e., the individual 3-minute

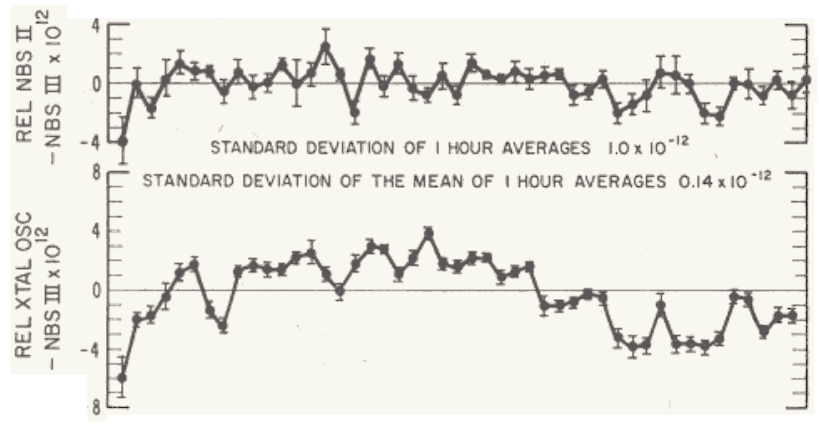

Fig. 7. 48-hour stability comparison between two cesium standards at the National Bureau of Standards (NBS II vs NBS III December 18-20, 1963)

averages behave as random samples from a stable probability distribution - these two estimates should be equal. In view of the reasonably good agreement observed we can predict, in a probability, sense, that for any similar 1-hour frequency measurement the uncertainty in the result produced by the random variations would be about $\pm 1 \times 10^{-12}$. Although, in principle, it is possible to reduce this uncertainty to an insignificant level by making the measurement time sufficiently long, it is normally impractical to do so.

The primary source of these random fluctuations is the shot noise from the beam itself. The process by which a fluctuation in beam current may result in a frequency fluctuation of the oscillator controlled by the atomic resonance may be thought of in the following way. The servo system, as we have noted pre- viously, responds to components of the detected beam current which are at or very near the modulation frequency. Such a component will normally occur only when the microwave frequency is not exactly equal to the cesium frequency. However, even if the microwave frequency is exactly tuned to the resonance peak, a beam fluctuation will have a frequency spectrum containing components at or near the modulation frequency. The servo system will interpret this component as a legitimate error signal and correct the oscillator accordingly, producing a momentary error in the controlled oscillator's frequency. The manner in which this resulting instability depends upon the more significant beam tube parameters may be seen from the much-simplified resonance line shape shown in

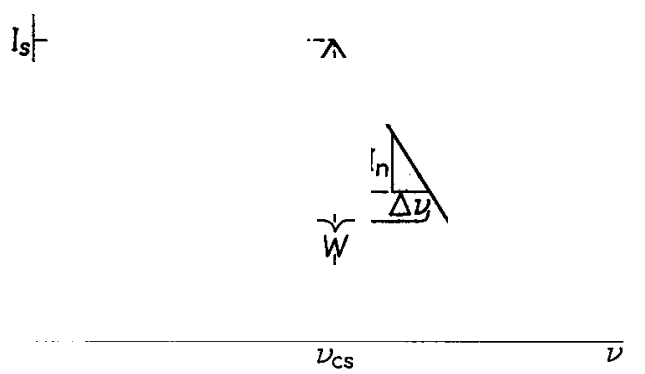

Fig. 8. Simplified resonance line shape for illustrating the relation between frequency fluotuations due to beam fluctuations and the signal-to-noise ratio and linewidth

Fig. 8. In this plot $I_{s}$ is the peak detected current at the resonance maximum, $W$ is the full resonance width where $I=I_{s} / 2, I_{n}$ represents a fluctuation of the beam current, and $\Delta v$ is the corresponding frequency fluctuation. Using the relation between the two similar triangles with sides $\Delta v$ and $I_{n}$ in one case and $W / 2$ and $I_{s} / 2$ in the other, we can write immediately:

$$
\begin{array}{ccc}
\frac{\Delta v}{I_{n}} & \frac{W / 2}{I_{s} / 2} & \frac{W}{I_{s}} \\
\frac{\Delta v}{v_{0}}=\frac{W}{v_{0}} & \frac{I_{n}}{I_{s}} .
\end{array}
$$

Now, if we make the reasonable assumption that the main source of beam fluctuations is shot noise, we can use the fact that $\overline{I_{n}^{2}} \propto B$, where $B$ is the bandwidth of the detection system by writing:

$$
\overline{I_{n}^{2}}=\varrho_{n o}^{2} B,
$$

where $\varrho_{n o}^{2}$ is the mean square noise current per unit bandwidth. From (24) and (25) we have,

$$
\left(\frac{\Delta v}{v_{0}}\right)_{R M S}=\frac{W}{v_{0}} \frac{\varrho^{n o}}{I_{s}} \sqrt{B} .
$$

Finally, since $B=1 / T$, where $T=$ the time over which the measurement is made, we get

$$
\left(\frac{\Delta v}{\nu_{0}}\right)_{R M S}=\frac{W}{\nu_{0}} \frac{Q_{n o}}{I_{d}} T^{-\frac{1}{2}} .
$$

This expression shows the advantages of a narrow resonance line, high signal-to-noise ratio, and long measuring time in minimizing frequency instability due to beam fluctuations. A more detailed consideration of the observed frequency instability would, of course, have to take into account the influence of the servo system corrections (CUTLER, 1964; KaRTASChoFf, 1964; Lacey, Helgesson, and Holloway, 
1964), but relation (27) is nevertheless very useful for comparing various beam-system designs.

Servo Errors. Systematic errors resulting from imperfections or misadjustments in the servo system used to develop a correction signal for electronically locking a quartz oscillator to the cesium resonance frequency also fit into this category. The main problem here is second harmonic distortion of the modulation signal which is usually chosen to be in the range $20-100 \mathrm{~Hz}$. The source of the distortion may be in the modulation oscillator itself or in the modulation circuitry due to non-linear response. The resulting frequency shift is

$$
\Delta \nu=1 / 2 D v_{d} \sin \alpha, \text { where }
$$

$100 D=$ percent second harmonic distortion,

$\nu_{d}=$ peak frequency deviation of the modulation, and

$\alpha=$ phase of the second harmonic relative to the fundamental.

Since the peak frequency deviation of the modulation is usually set to about one half of the resonance line other servo components, but, in general, these must be evaluated for each particular servo system.

Multiplier chains may also contribute errors if transient phase shifts, such as due to temperature variations, are present. This effect may cause the output frequency to depart from an exact integral multiple of the input for significant periods of time. Since it is actually the output microwave frequency that is maintained by the servo system in synchronism with the cesium resonance, the chain input frequency from the controlled oscillator will be in error in this case.

Most investigators have found no definite dependence of the frequency on the microwave power level over reasonable ranges of power both up and down from the level corresponding to optimum transition probability. However, the frequency shifts resulting from some of the other error sources may be power dependent.

\section{Combination of Errors}

The process of determining an accuracy figure for a particular standard then consists of evaluating by one means or another the extent to which contributions from the various sources mentioned above may affect the accuracy of frequency measurements. These individual contribu. tions must then be combined in some sense to arrive at an overall accuracy figure. Since the above contributions may reasonably be considered to be independent in the statistical sense, the combined uncertainty may be taken as the square root of the sum of the squares of the individual uncertainties.

An example of this procedure is summarized in Tab. 1, which gives the estimated individual contributions to inaccuracy for the newest NBS cesium standard, NBS III. The individual estimates given are subject to the following conditions:

(a) In most cases the estimates are considered to be reasonable limits of error, approximately corresponding to $3 \sigma$ estimates. Exceptions are the estimated uncertainties due to $\overline{\mathrm{H}(x)}$ (Item 1) and random errors (Item 10), which were determined by statistical analysis as $1 \sigma$ limits.

(b) The estimates involving $C$-field effects, cavity phase shift, spectrum of the excitation, and servo effects are rechecked periodically.

(c) The estimates involving first order Doppler shift and multiplier chain transients are considered somewhat tentative, at present, due to a lack of conclusive experimental evidence in these areas.

Present accuracy figures quoted for NBS, NPL, and Neuchatel are $\pm 5.6 \times 10^{-12}, \pm 3 \times 10^{-11}$, and $\pm 1 \times 10^{-11}$, respectively. There is some variation in the methods used to make these estimates.

\section{Cesium Standards in Operation}

Cesium standards presently in operation may be usefully classified as laboratory standards or commer- error-signal processing circuitry. Other errors are possible which are related to imperfections in certain 
cial standards. The laboratory standards are those instruments which are capable of independent evaluation by the controlled variation of the many operating parameters discussed above. They are intended for stationary rather than transportable use. Thus they may have more elaborate power supplies, vacuum systems, signal sources, environmental control, etc. The commercial standards have the objective of transportable use in varied environments, often under limitations of size, weight, and power consumption. They are characterized by fixed design as a result of prior laboratory research and development.

The design and performance characteristics of most of the laboratory and commercial standards are available from the references. Thus we have elected merely to tabulate a few of the characteristics in Tab. 2 and 3 for the sake of illustration.

Accuracy of a cesium beam frequency standard is enhanced by providing a small line width and a large signal to noise ratio, as previously discussed. The requirement of small line width calls for a long interaction length, but the requirement of good signal-tonoise ratio calls for a short beam length in order to enjoy large aperture beam optics.

The laboratory instruments have in general elected to favor small line width by using a long interaction length. They overcome the shot noise error by accepting sufficiently long averaging times according to (27).

The commercial instruments have been forced to short tubes by size limitations. They have tended to compensate in two ways. The first is greater use of velocity selection of slow atoms, and the second is use of larger aperture and more sophisticated beam optics to improve the signal-to-noise ratio. This has a further benefit of improved stability over short averaging times.

Intensive further development of the commercial standards is continuing with the objective of further

Table 2. Characteristics of Certain Laboratory Cesium Beam Standards

\begin{tabular}{|c|c|c|c|c|c|c|c|c|}
\hline Instrument & $\begin{array}{c}\text { Approximate } \\
\text { Date Of First } \\
\text { Operation }\end{array}$ & $\begin{array}{l}\text { Interaction } \\
\text { Length (cm) }\end{array}$ & $\begin{array}{c}\alpha^{\star} \\
\times 10-4 \\
\mathrm{~cm}-s^{-1}\end{array}$ & $\bar{v} / \alpha$ & $\begin{array}{l}\text { Observed } \\
\text { Line } \\
\text { Width }(\mathrm{Hz})\end{array}$ & Servo? & $\begin{array}{l}\text { Ascribed } \\
\text { Aceurncy } \\
\times 10^{11}\end{array}$ & Reference \\
\hline $\begin{array}{l}\text { NPL-I } \\
\quad(C \& 1)\end{array}$ & 1955 & 47 & 2.44 & 1 & 330 & No & \pm 100 & $\begin{array}{l}\text { EssEs and PARrY, } \\
\text { 1955; EssEx and } \\
\text { PAREX, } 1957 \text {. }\end{array}$ \\
\hline $\begin{array}{l}\text { NPL-1I, } \\
\text { Mod. } 1\end{array}$ & 1959 & 265 & 2.30 & 0.9 & 52 & No & \pm 3 & $\begin{array}{l}\text { Esser, Stemle, and } \\
\text { Surcliwre, } 1964 .\end{array}$ \\
\hline NBS.1 & 1959 & 55 & 2.3 & 1.1 & 300 & No & \pm 1 & $\begin{array}{l}\text { BeEHLER, ATKTNSON, } \\
\text { Hrim, and SNIDER, } \\
1962 \text {. }\end{array}$ \\
\hline NBS-II & 1960 & 164 & 2.3 & 1.3 & 120 & Yes & \pm 1 & $\begin{array}{l}\text { BexHink, ATKTNSos, } \\
\text { Hrim, and SxIDEH, } \\
1962 \text {. }\end{array}$ \\
\hline NBS-III & 1963 & 306 & 2.3 & 1.5 & 48 & Yes & \pm 0.6 & $\begin{array}{l}\text { BEEmLre and Grazs, } \\
1965 .\end{array}$ \\
\hline LSRH-I & 1959 & 100 & 2.16 & - & -9 & No & - & - \\
\hline LSRH-II & 1960 & 409 & 2.25 & $\begin{array}{l}0.7 \\
1.1\end{array}$ & $\begin{array}{l}20 \\
35 \\
42\end{array}$ & Yes & \pm 1 & 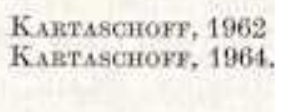 \\
\hline NRC-I & 1958 & 80 & - & - & 230 & No & 100 & $\begin{array}{l}\text { KaLes, BAI,WY, and } \\
\text { DAaMs, } 1959 .\end{array}$ \\
\hline
\end{tabular}

* $\alpha$ is the most probable velocity of the Maxwell-Boltzmann distribution in the oven.

Table 3. Characteristics of Certain Commercial Cesium Beam Standards

\begin{tabular}{|c|c|c|c|c|c|c|}
\hline $\begin{array}{l}\text { Instrument } \\
\text { Model No. }\end{array}$ & $\begin{array}{l}\text { Interaction } \\
\text { Length } \\
\text { (cm) }\end{array}$ & $\left|\begin{array}{c}\alpha \star \times 10^{-4} \\
\mathrm{~cm}-\mathrm{s}^{-1}\end{array}\right|$ & $\bar{v} / \alpha$ & $\begin{array}{c}\text { Observed } \\
\text { Linewidth }(\mathrm{Hz})\end{array}$ & $\begin{array}{c}\text { Ascribed } \\
\text { Accuracy } \\
\times 10^{11}\end{array}$ & Reference \\
\hline NC-1001 (National Company) & 94 & 2.1 & 0.86 & 125 & $\begin{array}{c}120 \\
(100)\end{array}$ & $\begin{array}{l}\text { REDER, } 1963 \\
\text { (data sheet) }\end{array}$ \\
\hline NC-1501 (National Company) & 24 & 2.2 & 0.64 & 375 & \pm 20 & \\
\hline NC-1601 (National Company) & 11 & 2.2 & 0.4 & 500 & $\begin{array}{c} \pm \mathbf{5 0} \\
\text { (data sheet) }\end{array}$ & \\
\hline NC-2001 (National Company) & 90 & 2.2 & 0.63 & 100 & 5 & , \\
\hline $\begin{array}{l}\text { HP-5060 A (Hewlett- } \\
\text { Packard Company) }\end{array}$ & 12.4 & - & - & 530 & \pm 2 & $\begin{array}{l}\text { BAGLEY and CoTLER, } \\
\text { 1964, Hewlett-Packard } \\
\text { Data Sheet }(8 / 6 / 64)\end{array}$ \\
\hline $\begin{array}{l}\text { P\& B-3120 (Pickard and } \\
\text { Burns Electronics) }\end{array}$ & - & - & - & 260 & \pm 5 & $\begin{array}{l}\text { PICKARD and Burns } \\
\text { Data Sheet } 124\end{array}$ \\
\hline
\end{tabular}

* $\alpha$ is the most probable velocity of the Maxwell-Boltzmann distribution in the oven. 
improving accuracy, stability, packaging, and resistance to environmental changes. Improvements of one or two orders of magnitude are sought. The reader is advised to search the current periodical and report literature for details.

\section{Comparison of Standards}

The intercomparison of independent cesium beam standards provides an important check upon the estimates of accuracy assigned to the standards and should certainly be taken into account in any final accuracy assignments.

Direct Comparison. If the standards are located within the same laboratory the comparison is usually straightforward and can be made with high precision. An example of this sort of comparison is the direct measurement of the difference frequency between NBS II and NBS III over a 48-hour period, the results of which are shown in Fig. 7. Repeated measurements of this type over an extended time have shown the average frequency difference between these two independent standards to be $3 \times 10^{-12}$, which is consistent with the quoted accuracies of $\pm 1 \times 10^{-11}$ and $\pm 5.6 \times 10^{-12}$ for NBS II and NBS III, respectively.

Frequency Comparison by Radio. In order to compare standards in different locations, such as the various primary standards of frequency maintained by the national standards laboratories, several different techniques have been employed. The most widely used technique is the simultaneous (or approximately so) measurement of one or more standard frequency transmissions by a number of different laboratories in terms of their own atomic standard. Analysis of these data yields values for the differences among the monitoring cesium standards, although uncertainties due to propagation effects usually limit the precision of this type of comparison to one or two parts in $10^{11}$, even when averages over many months are used. Best results are generally obtained for international ${ }^{*}$ comparisons when VLF transmissions, such as NBA $(24 \mathrm{kHz})$ and GBR $(16 \mathrm{kHz})$, are utilized.

Morgan, BLATR, and Crow (1965) have used a statistical analysis-of-variance technique on 18 months of VLF monitoring data from seven different laboratories in the United States, Europe, and Canada in order to separate the variance of the observations at each laboratory into three components: (a) long-term mean differences among the atomic standards; (b) effects of the fluctuations of the receiving system, propagation effects peculiar to the particular radio path, and measurement errors; and (c) fluctuations of the transmitter signals and propagation effects common to all the radio paths. They concluded that during the July 1961-December 1962 period considered the means of all seven atomic frequency standards (commercial and laboratory types) agreed with the grand mean to within $\pm 2 \times 10^{-10}$, while the means of the four laboratory-type standards agreed with their grand mean to within $\pm 1 \times 10^{-10}$. An indication of the components of measurement fluctuations as observed at each of seven different laboratories is given in Tab. 4 and 5 for GBR and NBA, respectively. The values of $x_{i}-\bar{x}$ are the deviations of the 18 month mean for each atomic standard from the 18 month grand mean. These figures give an idea of the degree to which agreement among independent standards was attained and maintained over 18 months. The " $\hat{\tau}$ average" values are essentially an average standard deviation associated with the day-to-day fluctuations of the transmitted signal plus propagation effects common to all radio paths. Similarly, the "Av. $\hat{a}_{i}$ " values represent average standard deviations associated with day-to-day fluctuations in the receiving system, including effects peculiar to the particular propagation path, measurement errors, as well as fluctuations in the local standard itself. Although the $\hat{\tau}$ and $\hat{a}_{i}$ are of comparable magnitude to the variations in the standards among each other $\left(x_{i}-\bar{x}\right)$, the statistical treatment enables all these effects to be separated and estimated.

Time Scale Comparison. Another technique which has been used very successfully for the comparison of atomic standards at remote installations involves the comparison of independent time scales based on each of the cesium standards. For example, the NBS-A atomic time scale and the $\mathrm{TA}_{1}$ atomic time scale based on the laboratory cesium standards at the National Bureau of Standards, Boulder, Colorado, USA and at the Laboratoire Suisse de Recherches Horlogeres,

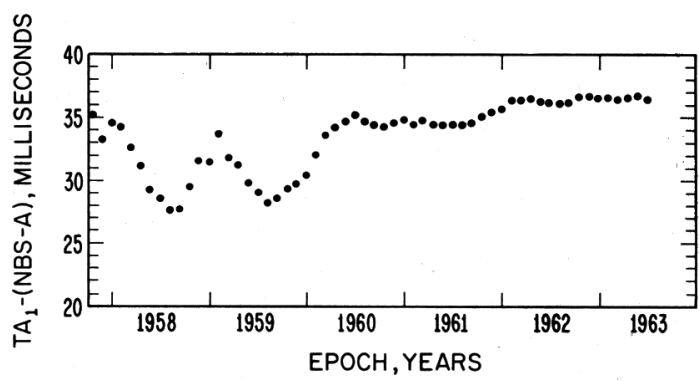

Fig. 9. Comparison of the atomic time scales $T A_{1}$ and NBS-A

Neuchatel, Switzerland, respectively, have been compared since 1958 (Bonanom, Kartaschoff, Newman, Barnes, and Atkinson, 1964). Fig. 9 is a plot of the difference between the time scales as a function of epoch, where the ordinate is the reception time of the WWV time signals at Neuchatel on the $\mathrm{TA}_{1}$ scale minus the emission time of the time signals on the NBS-A scale. Exact agreement of the two frequency standards would be indicated on this plot by a horizontal line. The obvious improvement occurring in 1960 was due to a change in standards at LSRH from an ammonia maser to a cesium standard and to the adoption of NBS II as the standard at NBS. The divergence of the scales equivalent to about $8 \times 10^{-11}$ occuring from 1961.6 to 1962.1 was due to a temporary magnetic shielding problem with the LSRH standard as reported by KaRTASCHOFF (KaRTASCHOFF, 1962). If the observed rate of divergence of the two time scales from 1960.5 - 1961.6 and since 1962.2 is attributed entirely to a systematic difference between the two cesium standards, a frequency difference of $1 \times 10^{-11}$ is indicated. This result is consistent with the quoted accuracy figures for both standards.

Portable Standards. Still another method of comparing two frequency standards at remote installations involves carrying a portable standard between the two laboratories, making frequency comparisons at both locations with the assumption that the portable 
'able 4. Errors in Terms of GBR Data and Indicated Standards $\hat{\tau}$ Average $^{\star}=1.26 \times 10^{-10}$

$\hat{a}_{i}$ Average $^{\star}$ per Station as follows:

\begin{tabular}{|c|c|c|c|c|}
\hline Station & $\left(x_{i}\right.$ & $\bar{x}) \quad 10^{10}$ & $\mathrm{Av} \cdot \hat{a}_{\boldsymbol{i}}$ & $\begin{array}{l}\text { No. Days } \\
\text { Observed }\end{array}$ \\
\hline CNET & & 1.68 & 1.05 & 278 \\
\hline CRUFT & & 0.78 & 0.41 & 136 \\
\hline LSRH & & 1.04 & 0.39 & 244 \\
\hline NBS & & 0.68 & 0.66 & 244 \\
\hline NOB & & 0.34 & 0.62 & 278 \\
\hline NPL & & .0 .05 & 1.00 & 278 \\
\hline NRC & & .0 .65 & 1.97 & 222 \\
\hline
\end{tabular}

* RMS weighted Average

\section{Station Indentifications}

CNET Centre National d'Etudes des Telecommunications, Bagneux, Seine, France (Commercial standard).

GRUFT Cruft Laboratories, Harvard University, Cambridge, Massachusetts, USA (Commercial standard).

LSRH Laboratoire Suisse de Recherches Horlogeres, Neuchatel, Switzerland (Laboratory standard).

NBS National Bureau of Standards, Boulder, Colorado, USA (Laboratory standard).

NOB U. S. Naval Observatory, Washington, D. C., USA (Radio average of 9 standards).

NPL National Physical Laboratory, Teddington, Middlesex, England (Laboratory standard).

NRC National Research Council, Ottawa, Ontario, Canada (Laboratory standard).

Table 5. Errors in Terms of NBA Data and Indicated Standards $\hat{\tau}$ Average $^{\star}=0.68 \times 10^{-10}$

$\hat{a}_{i}$ Average $^{\star}$ per Station as follows:

\begin{tabular}{lrc|cc}
\hline Station & $\left(x_{i}\right.$ & $10^{10}$ & Av. $\hat{a}_{i} \times 10^{10}$ & $\begin{array}{c}\text { No. Days } \\
\text { Observed }\end{array}$ \\
\hline CNET & 1.89 & 0.95 & 197 \\
CRUFT & .1 .05 & 1.13 & 131 \\
LSRH & .0 .99 & 0.63 & 197 \\
NBS & .0 .66 & 0.63 & 161 \\
NOB & 0.27 & 0.51 & 197 \\
NPL & 0.28 & 1.24 & 102 \\
NRC & -1.30 & 1.82 & 113
\end{tabular}

* RMS weighted Average

\section{Station Indentifications}

CNET Centre National d'Etudes des Telecommunications, Bagneux, Seine, France (commercial standard).

CRUFT Cruft Laboratories, Harvard University, Cambridge, Massachusetts, USA (commercial standard).

ISRH. Laboratoire Suisse de Recherches Horlogeres, Neuchatel, Switzerland (laboratory standard).

NBS National Bureau of Standards, Boulder, Colorado, USA (laboratory standard).

NOB U.S. Naval Observatory, Washington, D. C., USA (commercial standard).

NPL National Physical Laboratory, Teddington, Middlesex, England (laboratory standard).

NRC National Research Counoil, Ottawa, Ontario, Canada (laboratory standard).

standard's frequency remains stable between laboratories. This type of measurement has been made feasible by the recent development of highly-stable portable commercial cesium and rubidium standards. In June 1964, two commercial cesium standards which had been taken to Switzerland for an instrument exhibition were compared with the long cesium standard at LSRH in Neuchatel (BAGLEY and CUTLER, 1964). Four days later the commercial instruments were measured in terms of the NBS standard in
Boulder, Colorado, after being kept in continuous operation during the intervening time. The results of these measurements again showed the Swiss and U. S. standards to be in agreement to better than $1 \times 10^{-11}$. The assumption of perfect stability of the commercial standards between comparisons did not appear to limit the measurements in view of the excellent longterm stability demonstrated by these instruments during one week's continuous operation in Switzerland.

\section{Atomic Time Standards}

Construction of Atomic Time Scales. An atomic time scale based upon the cesium transition can be constructed in several different ways. Basically, one needs to have some means of accumulating cycles of the periodic phenomenon (in this case, the cesium transition frequency) providing the unit from an arbitrarily chosen origin. Since most cesium standards employ a quartz-crystal oscillator which is electronically locked to the cesium resonance, the most direct method would be to divide the quartz oscillator frequency down to a suitable value for driving a clock directly. Because of the practical difficulty in keeping a complex system such as a cesium standard in continuous operation, however, an indirect technique has usually been used in which the clock is driven by a free-running quartz oscillator of nominal frequency $\nu_{n}$ which is periodically calibrated in terms of the cesium standard to give its actual frequency $v_{a}$. These calibrations are then used to convert the sufficiently short indicated quartz time $\Delta \tau$ to atomic time $\Delta t$ according to the relation $\Delta t=\left(v_{n} / \nu_{a}\right) \Delta \tau$ with the assumption that the oscillator frequency has drifted linearly between calibration points. The interval between calibrations ranges from 1 day for the A.1 (see below) and NBS-A scales to 10 days for the $\mathrm{TA}_{1}$ scale. Errors introduced by departure of the actual oscillator drift from linearity as assumed, due to inherent oscillator noise and other causes, have been studied by a number of people (for example, DE PrINs, 1961).

Particular Time Scales. The various atomic time scales presently in use differ essentially only in the particular cesium standard or standards chosen for the basis of the scale. Thus, the A.1 atomic time scale developed at the United States Naval Observatory in 1958 is based upon a weighted average of 9 different laboratory and commercial cesium standards located in various laboratories throughout the world (MARKoWITZ, 1962 IRE). The published daily measurements of certain standard frequency transmissions, such as NBA and GBR, in terms of the various cesium standards are utilized to determine the frequency of the A.1 master oscillator in terms of the weighted average. The origin of the A.1 scale is chosen to be at $0^{\mathrm{h}} 0^{\mathrm{m}} 0^{\mathrm{s}}$ on the UT 2 time scale on 1 January 1958*.

The $\mathrm{TA}_{1}$ scale established in Switzerland by the Neuchatel Observatory is based upon the cesium resonance frequency as indicated by the cesium standard at the Laboratoire Suisse de Recherches Horlogeres (since 1960) (BoNanomI, KaRTASCHOFF, NEWMAN,

* UT 2 is one of the Universal Time scales based on the rotation of the earth. Its rate is a measure of the mean speed of rotation of the earth with known periodic variations remov. ed. UT 2 is still not uniform time, however, because of progressive changes in the speed of rotation of the earth. 
Barnes, and Atknnson, 1964). The origin of this scale was set to coincide with Ephemeris Time at $0^{\mathrm{h}} 0^{\mathrm{m}} 0^{\mathrm{s}}$ UT 2 on 1 January 1958*.

Similarly, the NBS-A atomic time scale is based upon the United States Frequency Standard (USFS) maintained at the National Bureau of Standards, Boulder, Colorado, USA.

The epoch of this scale was set to be approximately equal to that of UT 2 at $0^{\mathrm{h}} 0^{\mathrm{m}} 0^{\mathrm{s}}$ on 1 January 1958. The NBS-UA scale is also based on the USFS but the frequency offsets and step adjustments in epoch as announced by the Bureau International de l'Heure in Paris are incorporated, resulting in an interpolated "universal" time scale based on an atomic time scale.

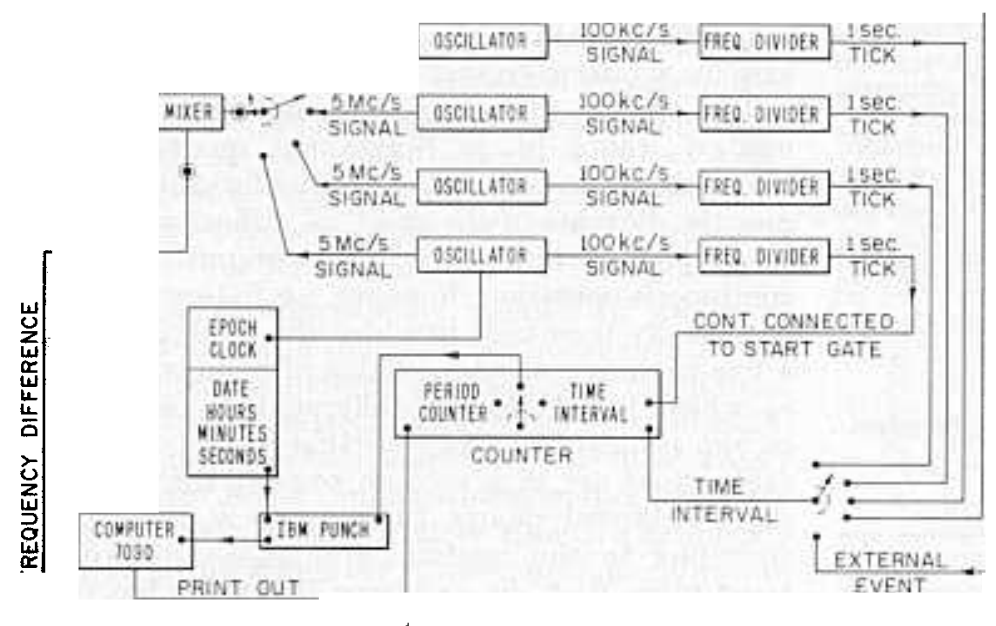

Fig. 10. Block diagram of the NBS-A atomic time scal

A block diagram of the components of the NBS-A scale is shown in Fig. 10. Each of the 5 oscillator-clock combinations operates independently of all others and a weighted average constitutes the time scale. A detailed description of the construction, operation, and results of the NBS-A and NBS-UA scales will be published (BARNES, 1965).

Synchronization of Clocks. A problem of interest involves the synchronization of clocks at remote locations. Interesting and novel techniques have been utilized during recent years for this purpose, including clock carrying experiments, the use of Loran- $C$ transmissions and artificial earth satellites. In $1959-1960$ the U.S. Army Signal Corp first demonstrated the feasibility of using airborne atomic clocks to obtain synchronization of widely separated clocks to within $5 \mu$ sec (REDER, Brown, WINKLER, and Bickart, 1961). Extensive use of portable, high-precision quartz clocks has been made by NBS personnel for synchronizing clocks at the Boulder, Colorado laboratories and at the WWV transmitter site in Maryland (BARNES and Fry, 1963). Uncertainties in the initial synchronization due to the portable clock were within $5 \mu$ sec. Later checks on this synchronization by means of further clock-carrying trips between Boulder and the WWV site were made with measurement accuracies approaching $1 \mu \mathrm{sec}$. In June 1964 , in connection with

* Ephemeris Time is defined by the orbital motion of the earth about the sun and is determined, in practice, from observations of the orbital motion of the moon about the earth.

\section{Summary}

Any acceptable standard of time must satisfy the following requirements: continuity of operation, generation of a unit which remains constant with respect to other acceptable measures of time, accuracy greater than or equal to standards based on other definitions, accessibility to all who need it, a characteristic period of convenient size, and a capability for accumulating the units to give epoch. Although astronomical standards based on the observed positions of celestial bodies meet these requirements very well, atomic standards have been developed since 1945 which possess a much higher accuracy with respect to the definition and greatly improved precision, making it possible to provide better results in a much shorter averaging time. Various types of atomic standards have been developed and evaluated, including ammonia absorption cells, ammonia and atomic hydrogen masers, rubidium gas cell devices, and a,tomic beam devices using cesium and thallium. Cesium standards are the most highly developed at present with accuracies of $\pm 1 \times 10^{-11}$ having been achieved in several different laboratories.

Although there is no universal agreement on how to specify the performance of cesium standards, various estimates of accuracy, precision, stability, and reproducibility are often used. The specification of accuracy requires an appropriate combination of random and systematic components of error. These various components have been thoroughly analyzed both theoretically and experimentally for cesium by many different laboratories and commercial firms.

Different cesium standards have been compared both directly within a given laboratory and indirectly between remote locations bv means of standard frenuence and time. 
radio transmissions, comparisons of independent time scales constructed from the standards, and the carrying of portable clocks between the remote locations. Results show that frequency differences are within $\pm 2 \times 10^{-10}$ for all standards and $\pm 1 \times 10^{-10}$ for the laboratory-type standards.

A number of independent atomic time scales have been constructed based on either a group of cesium standards (A. 1) or on a particular standard (TA 1 , NBS-A, NBS-UA). Clocks based on these time scales have been synchronized over intercontinental distances by clock-carrying experiments, use of radio transmissions, and use of artificial earth satellites. Accuracies of the order of 1 microsecond have been achieved.

\section{References \\ 1930 - 1954}

[1] Conen, V. W.: The nuclear spin of cesium. Phys. Rev. 46, 713 (1934).

[2] DICKE, R. J.: The effect of collisions upon the Doppler width of spectral lines. Phys. Rev. 89, 472 (1953).

[3] Frrim, E.: Ưber die magnetischen Momente der Atomkerne, Z. Physik 60, 320 (1930).

[4] -, and E. SEgRé: Zur Theorie der Hyperfeinstruktur. Z. Physik 82, 729 (1933).

[5] Fraster, R. G. J.: Molecular rays. Cambridge, England: Cambridge University Press 1931.

[6] Fraser, R.: Molecular beams. London: Metheun and Company 1937.

[7] Gordon, J. P., J. H. Zeiger, and C. H. Townes, Molecular microwave oscillator and new hyperfine structure in the microwave spectrum of $\mathrm{NH}_{\mathbf{3}}$. Phys. Rev. 95, 282 (1954).

[8] Hershberger, W. D., and L. E. Norton, Frequency stabilization with microwave spectral lines. R C A Rev. 9, 38 (1948).

[9] Kellogg, J. B. M., and S. MillmaN: The molecular beam magnetic resonance method. The radio frequency spectra of atoms and molecules. Rev. Mod. Phys. 18, 323 (1946).

[10] Kopfermann, H.: Nuclear moments, E. E. Schnetder, translator, and H. S. W. MASSEY, editor, Pure and Appl. Phys. Ser., Vol. 2, p. 114ff. New York: Academic Press 1958.

[11] Lyons, H.: Spectral lines as frequency standards. Ann. N. Y. Acad. Sci. 55, 831 (1952).

[12] RAMSEY, N.: A molecular beam resonance method with separated oscillating fields. Phys. Rev. 78, 695 (1950).

\section{5}

[13] Basov, N. G., and A. M. Prokronov: The molecular oscillator and amplifier. Uspekhi Fiz. Nauk. 57, 485 (1955).

[14] Essen, L., and J. V. L. PArry: An atomic standard of frequency and time interval. Nature 176, 280 (1955).

[15] SмIтH, K. F.: Molecular beams, London: Metheun and Company, and New York: John Wiley and Sons, Inc. 1955.

[16] Zacharias, J. R., J. G. Yates, and R. D. HaUn: An atomic frequency standard. Proc. I. R. E. 43, 364 (1955).

\section{6}

[17] Datz, S., and E. TAYLOR: Ionization on platinum and tungsten surfaces. I. The alkali metals. J. Chem. Phys. 25, 389 (1956). II. The potassium halides. J. Chem. Phys. 25, 395 (1956).

[18] Essen, L., and J. V. L. Parry: Atomic and astronomical time. Nature 177, 744 (1956).

[19] Ramsey, N. F.: Molecular beams. Oxford, England: Oxford University Press 1956.

[20] WitTKe, J. P., and R. H. Dicke: Redetermination of the hyperfine splitting in the ground state of atomic hydrogen. Phys. Rev, 103, 620 (1956).

1957

[21] Essen, L., and J. Parry: The caesium resonator as a standard of frequency and time. Phil. Trans. Roy. Soc. London 250, 45 (1957).

[22] HaUN, R. D., Jr., and J. R. ZaCharias: Stark effect on Cs-133 hyperfine structure. Phys. Rev. 107, 107 (1957).

[23] KastLER, A.: Optical methods of atomic orientation and of magnetic resonance. J. Opt. Soc. Am. 47, 460 (1957)

[24] LYoNs, H.: Atomic clocks. Sci. American. 196, 71 (1957).
[25] Pizroe, J. A.: Intercontinental frequency comparison by very low frequency radio transmission. Proc. I. R. E. 45, 794 (1957)

\section{8}

[26] Essen, L., J. V. L. Parky, W. Markowitz, and R. G. HALL: Variation in the speed of rotation of the earth since June 1955. Nature 181, 1054 (1958).

[27] $\rightarrow$, J. H. Holloway, W. A. Matnberger, F. H. REDER, and G. M. R. WINkLER: Comparison of caesium frequency standards of different construction. Nature 182, 41 (1958).

[28] Kalra, S. N., R. Bamey, and H. Daams: Cesium beam standard of frequency. Can. J. Phys. 36, 1442 (1958).

[29] Markowitz, W., R. G. Hall, L. Essen, and J. V. L. PARRY: Frequency of cesium in terms of ephemeris time. Phys. Rev. Letters I, 105 (1958).

[30] MoCoubrex, A. O.: Results of the comparison: Atomichron-British cesium beam standard. IRE Trans. on Instr. I-7, 203, (1958).

[31] MockLER, R. C.: Etalons de temp atomiques et moleculaires au National Bureau of Standards, in proces verbaux des seamces, rapport et annexes, 1re session (1957), Comité Consultatif pour la Définition de la Seconde, pp. 38 - 42. (Comité International des Poids et Mesures.) Proces-Verbaux des Seances, 2. serie, tome 26-B. Paris: Gauthier-Villars 1958.

1959

[32] ANDres, J. M., D. J. FARMer, and F. T. Ionouxe: Design studies for a rubidium gas cell frequency standard. IRE Trans. on Military Electronics MIL-3, 178 (1959).

[33] Essen, L., E. G. Hope, and J. V. L. Parry: Circuits employed in the NPL caesium standard. Proc. Inst. Elec. Engrs. (London), Pt. B 106, 240 (1959).

[34] 一, and J. V. L. PARRY: An improved caesium frequency and time standard. Nature 184, 1791 (1959).

[35] Recent research in molecular beams, I. EstermanN, editor, New York: Academic Press, Jne. 1959.

[36] Holloway, J., W. Mainberger, F. H. Reder, G. M. R. WinkLer, L. Essen, and J. V. L. PARry: Comparison and evaluation of cesium atomic beam frequency standards. Proc. I. R. E. 47, 1730 (1959).

[37] KaLRA, S. N., R. BaILeY, and H. DaAms: Canadian cesium beam standard of frequency. Nature 183, 575 (1959).

[38] -, C. F. Pattenson, and M. M. Thompson: Canadian standard of frequency. Can. J. Phys. 37, 10 (1959).

[39] Kusch, P., and V. W. HugHes: Atomio and molecular beam spectroscopy. Encyclopedia of Physics, p. 1, S. FLÜGGE, editor. Berlin-Göttingen-Heidelberg: Springer 1959.

[40] MoCotrReY, A. O.: National's militarized cesium beam frequency standards. Proceedings of the 13th Annual Symposium on Frequency Control (U.S. Army Signal Research and Development Laboratory, Ft. Monmouth, N. J.) 276 (1959).

[41] Ramsey, N. F.: Shapes of molecular beam resonances Recent Researeh on Molecular Beams, I. EstkrmaNN, editor. New York: Academic Press, Inc. 1959.

[42] ZaNDBERG, E., and N. Ionov: Surface ionization. Uspekhi Fiz. Nauk. 57, 581 (1959); English Translation: Soviet Phys.-Usp. 2, 255 (1959).

\section{0}

[43] Barnes, J. A., and R. C. Mockler: The power spectrum and its importance in precise frequency measurements. IRE Trans. on Instr. I-9, 149 (1960).

[44] BeeHLer, R. E., R. C. Mockler, and C. S. SNider: A comparison of atomic beam frequency standards. Nature 187, $681(1960)$.

[45] Carpenter, R. J., E. C. Beaty, P. L. Bender, S. Saito, and R. O. Srone: A prototype rubidium vapor frequency standard. IRE Trans. on Instr. I-9 132, (1960).

[46] DePrins, J., and P. KartaschofF: Applications de la spectroscopie hertzienne a la mesure de frequences et du temps. Neuchatel, Switzerland: Laboratory Suisse Recherches Horlogeres 1960.

[47] Doherty, R., G. Hefley, and R. ItNhierd: Timing potential of Loran-C, Proceedings of the 14th Annual Symposium on Frequency Control (U. S. Army Signal Research and Development Laboratory, Ft. Monmouth, N. J.) 276 (1960). 
[48] Goldenberg, H. M., D. KLePPNer, and N. F. RAMSEY: Atomic hydrogen maser. Phys. Rev. Letters 5, 361 (1960).

[49] KaLRA, S. N., and R. BAITY: Experimental investigation of atomic beam resonance technique as applied to cesium clock, Quantum Electronics: A Symposium, p. 121. C. H. Townes, editor. New York: Columbia University Press 1960.

[50] Kartaschowf, P., J. Bonanomi, and J. DhPrins: Description of a long cesium beam frequency standard, Proceedings of the 14th Annual Symposium on Frequency Control (U. S. Army Signal Research and Development Laboratory, Ft. Monmouth, N. J.) 354 (1960).

[51] MoCodbreY, A.: Missileborn Atomichron frequency standard, Proceedings of the 14th Annual Symposium on Frequency Control (U. S. Army Signal Research and Development Laboratory, Ft. Monmouth, N. J.) $315(1960)$

[52] MERRIIL, F. G.: Frequency and time standards. A status report. IRE Trans. on Instr. I-9, 117 (1960).

[53] Mockler, R. C., R. E. BeEHLER, and J. A. Barnes: An evaluation of a cesium beam frequency standard. Quantum Electronics, A Symposium, C. H. Townes, editor, p. 127. (Columbia University Press, New York, N. Y.:1960).

[54] - - and C. S. SNIDFr: Atomic beam frequency standards. IRE Trans. on Instr. I-9, 120 (1960).

[55] - NBS atomic frequency standards, Proceedings of the 14th Annual Symposium on Frequency Control (U. S. Army Signal Research and Development Laboratory, Ft. Monmouth, N. J.) 298 (1960)

[56] - Atomic beam frequency standards, Proceedings of the 1960 Brookhaven Conference on Molecular Beams. New York: Brookhaven National Laboratory, Upton, L. I., 1960.

[57] Prarce, J. A.: The GBR experiment: a transatlantic frequency comparison between caesium-controlled oscillators. Proceedings of the 14th Annual Symposium on Frequency Control (U.S. Army Signal Research and Development Laboratory, Ft. Monmouth N. J.) 267 (1960).

[58] WINkLER, G.: A superior atomic clock for continuous long time operation. Proceedings of the 14 th Annual Symposium on Frequency Control (U. S. Army Signal Research and Development Laboratory, Ft. Monmouth, N. J.) 261 (1960).

\section{1}

[59] Azdrm, M., and T. Carver: Pressure, light and temperature shifts in optical detection 0-0 hyperfine resonance of alkali metals. Phys. Rev. 124, 800 (1961).

[60] Barnes, J. A., and L. E. Heim: A high-resolution ammonia maser spectrum analyser. IRE Trans. on Instr. I-10, 4 (1961).

[61] DrPRTNs, J.: Applications des masers $A \mathrm{~N}^{15} \mathrm{H}_{3}$ à la mesure et à la definition du temps. Unpublished, Université Libre de Bruxelles: Dootor of Sciences thesis 1961.

[62] McCouBREY, A. O.: Frequency control by microwave atomic resonance. Microwave Jl. 4, 65 (1961).

[63] MockLER, R. C.: Atomic beam frequency standards, Advances in Electronics and Electron Physics, Vol. 15, p. 1. L. Marton, editor. New York, N. Y.: Academic Press, Inc. 1961.

[64] Rarity, J., L. Saporta, and G. Weiss: The effects of frequency multipliers on the uncertainty of a frequency measurement. Proceedings of the 15th Annual Symposium on Frequency Control (U. S. Army Signal Research and Development Laboratory, Ft. Monmouth, N. J.) 261 (1961).

[65] Reder, F., P. Brown, G. Winkaler, and C. Bickart: Final results of a world-wide clock synchronization experiment (Project WOSAC). Proceedings of the 15th Annual Frequency Control Symposium (U.S. Army Signal Research and Development Laboratory, Ft. Monmouth, N. J.) pp. 226 (1961).

\section{2}

[66] Barnes, J. A., D. W. Allan, and A. E. WatNWright: The ammonia beam maser as a standard of frequency. IRE Trans. on Instr. I-11, 26 (1962).
[67] Brehler, R. E., W. R. Atkinson, L. E. Herm, and C. S. SNIDER: A comparison of direct and servo methods for utilizing cesium beam resonators as frequency standards. IRE Trans. on Instr. I-11, 231 (1962).

[68] Bonanom, J.: A thallium beam frequency standard. IRE Trans. on Instr. I-11, 212 (1962).

[69] DePrTns, J.: $\mathrm{N}^{16} \mathrm{H}_{3}$ double beam maser as a primary frequency standard. IRE Trans. on Instr. I-11, 200 (1962).

[70] Essen, L., and J. McA. Steele: The international comparison of atomic standards of time and frequency. Proc. Inst. Elec. Engrs. (London) Pt. B 41, 109 (1962).

[71] Georae, J.: Recent advances in cesium beam technology and characteristics of Rabi and Ramsey cesium beam tubes 17 inches long. IRE Trans. on Instr. I-11, 250 (1962).

[72] KARTASCHOFF, P.: Operation and improvement of a cesium beam standard having 4-meter interaction length. IRE Trans. on Instr. I-11, 224 (1962).

[73] KLEPPNER, D., H. M. Gold ENBERG, and N. F. RAMSEX: Theory of the hydrogen maser. Phys. Rev. 126, 603 (1962).

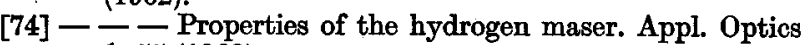
$1,55(1962)$

[75] Markowitz, W.: The atomic time scale. IRE Trans. on Instr. I-11, 239 (1962).

[76] - Time measurement technique in the microsecond region. Engineers Digest 135, 9 (1962).

[77] Muzushrma, M.: Theory of resonance frequency shifts due to the radiation field. Proceedings of the 16th Annual Symposium on Erequency Control (U.S. Army Signal Research and Development Laboratory, Ft. Monmouth, N. J.) 267 (1962).

[78] Packard, M. E., and B. E. Swartz: The optically pum. ped rubidium vapor frequency standard. IRE Trans. on Instr. I-11, 215 (1962).

[79] Richardson, J. M., R. E. Bexhler, R. C. MockLer, and R. L. FEY: Les étalons atomiques de frequence au NBS. Comité Consultatif pour la definition de la Seconde aupres du Comité International des Poides et Mesures, 2- session - 1961, p. 57. Paris: GauthierVillars and C-- 1962.

[80] Srmmoda, K.: Ammonia masers. IRE Trans. on Instr. I-11, 195 (1962).

[81] Stone, R. R., Jr.: Synchronization of local frequency standards with VLF transmissions. Proceedings of the 16th Annual Symposium on Frequency Control (U. S. Army Signal Research and Development Laboratory, Ft. Monmouth, N. J.) 227, (1962).

[82] Vessot, R. F. C., and H. E. Peters: Design and performance of an atomic hydrogen maser. IRE Trans. on Instr. I-11, 183 (1962).

\section{3}

[83] BARnes, J. A., and R. L. FeY: Synchronization of two remote atomic time scales. Proc. IEEE 51, 1665 (1963).

[84] BEEHLER, R. E., and D. J. GLAZE: Experimental evalua. tion of a thallium beam frequency standard. Proceedings of the 17th Annual Symposium on Frequency Control (U. S. Army Research and Development Laboratory, Ft. Monmouth, N. J.) 392 (1963).

[85] Crampton, S., D. Kueppner, and N. F. Ramsey: Hyperfine separation of ground state atomic hydrogen. Phys. Rev. Letters 11, 338 (1963).

[86] FARmre, D. J.: Performance and application of gas cell frequency standards. Proceedings of the 17th Annual Symposium on Frequency Control (U. S. Army Signal Research and Development Laboratory, Ft. Monmouth, N. J.) 449 (1963).

[87] Grorar, J.: Development and performance of a miniaturized cesium beam tube. Proceedings of the 17th Annual Symposium on Frequency Control (U.S. Army Signal Research and Development Laboratory, Ft. Monmouth, N. J.) 438 (1963).

[88] GUY, H. D.: Problems of frequency multiplication in atomic standards. Proceedings of the 17th Annual Symposium on Frequency Control (U. S. Army Signal Research and Development Laboratory, Ft. Monmouth, N. J.) 482 (1963).

[89] MockLeR, R. C.: Atomic frequency and time interval standards in the United States (1960 - 1963). Report to URSI U.S. National Committee-Commission I, February 8, 1963. 
[90] Newman, J., L. Fey, and W. R. Atkrnson: A comparison of two independent atomic tome scales. Proc. IEEE 51,498 (1963).

[91] REDER, F. H.: Achievements and problem areas of atomic frequency control. Proceedings of the 17th Annual Symposium on Frequency Control (U. S. Army Signal Research and Development Laboratory, Ft. Monmouth, N. J.) 329 (1963).

[92] Shirley, J. H.: Some causes of resonant frequency shifts in atomic beam machines. I. Shifts due to other frequencies of excitation. J. Appl. Phys. 34, 783 (1963).

[93] - Some causes of resonant frequency shifts in atomic beam machines. II. The effect of slow frequency modulation on the Ramsey line shape. J. Appl. Phys. 34, 789 (1963).

\section{4}

[94] BAGLEY, A. S., and L. S. CUThER: A modern solid-state, portable cesium beam frequency standard. Proceedings of the 18th Annual Symposium on Frequency Control (U.S. Army Signal Research and Development Laboratory, Ft. Monmouth, N. J.) 344 (1964).

[95] - A new performance of the flying clock experiment. Hewlett-Packard Company Journal 15, No. 1, (July 1964).

[96] Bonanom, J.: Les horlogues atomiques. Proceedings of the International Conference on Chronometry, Lausanne, 227 (1964).

[97] -, P. Kartaschoff, J. Newman, J. A. Barnes, and W. R. AtKInson: $A$ comparison of the $\mathrm{TA}_{1}$ and the NBSA atomic time scales. Proc. IEEE 52, 439 (1964).

[98] Brandemberger, J. H., J. Mtchate, P. Kartaschoff, and J. RACINE: Horlogue atomique avec tube à césium. Proceedings of the International Conference on Chronometry, Lausanne, 353 (1964).

[99] CUTLRR, L. S.: Some aspects of the theory and measurement of frequency fluctuations in frequency standards. Interim Proceedings of the Symposium on the Definition and Measurement of Short Term Frequency Stability (Goddard Space Flight Center, Greenbelt, Maryland) II-73 (1964).

[100] Essen, L. J., J. McA. Stekle, and D. Sutcliffe: The NPL frequency standard. Proceedings of the 18th Annual Symposium on Frequency Control (U. S. Army Signal Research and Development Laboratory, Ft. Monmouth, N. J.) 308 (1964).

[101] George, J., E. Wunderer, and T. Athanis: Recent progress in cesium beams at National. Proceedings of the 18th Annual Symposium on Frequency Control (U. S. Army Signal Research and Development Laboratory, Ft. Monmouth, N. J.) 322 (1964).

[102] Holloway, J. H., and R. F. LACEX: Factors which limit the accuracy of cesium atomic beam frequency standards. Proceedings of the International Conference on Chronometry, Lausanne, 317 (1964).
[103] -, and R. H. Woodward: Progress in the development of a cesium beam oscillator for aerospace guidance. Proceedings of the 18th Annual Symposium on Frequency Control (U.S. Army Signal Research and Development Laboratory, Ft. Monmouth, N. J.) 366 (1964).

[104] KaRTaschoff, P.: Etude d'un étalon de fréquence a jet atomique de césium. Unpublished. Docteur Es Sciences Techniques thesis. Zürich: L'ecole Polytechnique Fédérale 1964

[105] - Shot efiect influence on the frequency of an oscillator locked to an atomic beam resonator. Interim Proceedings of the Symposium on the Definition and Measurement of Short Term Frequency Stability (Goddard Space Flight Center, Greenbelt, Maryland) A-19 (1964).

[106] LACEY, R. F., J. H. HollowaY, and A. L. HeLgesson: Short term stability of passive atomic frequency standards. Interim Proceedings of the Symposium on the Definition and Measurement of Short Term Fre. quency Stability (Goddard Space Flight Center, Greenbelt, Maryland) III-3 (1964).

[107] MarkowITz, W.: High precision frequency and clock synchronization techniques on an international basis. Proceedings of the 18th Annual Symposium on Frequency Control (U. S. Army Signal Research and Development Laboratory, Ft. Monmouth, N. J.) 251 (1964).

[108] Menoud, C., J. Ractne, and P. Kartasohofr. Maser a hydrogène atomique. Quantum Electronics, p. 433. GrIVET, P., and N. BlokmberGEN, editors, New York: Columbia University Press 1964.

[109] MockLer, R. C.: Atomic frequency and time interval standards. URSI National Committee Report, XIV General Assembly Tokyo, September 1963, Commission I. Radio Science Journal of Research, Vol. $68 \mathrm{D}$, No. 5, May 1964.

[110] Pickard and Burns Electronics, Data Sheet Number 24.

\section{5}

[111] Barnes, J. A., and D. W. Allan: Private communication 1965 .

[112] Brefler, R. E., and D. J. Glaze: Private communication 1965.

[113] Morgan, A. H., B. E. Blatr, and E. L. Crow: Private communication 1965.

[114] RaMsiy, N. F.: The atomic hydrogen maser. Metrologia 1, 7 (1965).

R. E. BEEHLER

National Bureau of Standards

Atomic Frequency and Time

Interval Standards

Radio Standards Laboratory

Boulder, Colorado

\section{LETTERS TO THE EDITOR}

Division of Applied Physics, National Research Council, Ottawa, Canada

\section{Variation on the Paired Source Method of Measuring Dead Time}

By

\section{A. P. Baerg}

(Received April 5, 1965)

With 1 Figure in the Text

A number of inherently accurate methods for measuring the overall dead time of a radiation detection system are available. These include the decaying source method [1] and the commonly used paired source techniques [2]. Both of these, however, are based on the assumption that the dead time is nonextending (independent of count rate). Several more elaborate methods, using a series of individual sources [3] or source pairs [4], have been developed for measuring count rate dependent dead times. Some of these methods have recently been discussed in detail by $K_{R B P}$ [5] and KIRBY and BRAUN [6].

One difficulty in using the paired source method is that of ensuring reproducible geometry for each 